University of Nebraska - Lincoln

DigitalCommons@University of Nebraska - Lincoln

Papers in the Earth and Atmospheric Sciences

Earth and Atmospheric Sciences, Department

1986

\title{
A Possible Mechanism for the Diurnal Oscillations of Tropical Cyclones
}

Jay S. Hobgood

Follow this and additional works at: https://digitalcommons.unl.edu/geosciencefacpub

Part of the Earth Sciences Commons

This Article is brought to you for free and open access by the Earth and Atmospheric Sciences, Department of at DigitalCommons@University of Nebraska - Lincoln. It has been accepted for inclusion in Papers in the Earth and Atmospheric Sciences by an authorized administrator of DigitalCommons@University of Nebraska - Lincoln. 


\title{
A Possible Mechanism for the Diurnal Oscillations of Tropical Cyclones
}

\author{
JAY S. HOBGOOD \\ Department of Geography, University of Nebraska-Lincoln, Lincoln, NE 68588
}

(Manuscript received 7 March 1985, in final form 9 June 1986)

\begin{abstract}
A possible mechanism for the diurnal oscillations of tropical cyclones is presented. In the conceptual model developed to explain these features, the diurnal cycle of net radiation at the cloud tops is identified as the primary cause of the oscillations. Radiative cooling of the cloud tops at night steepens the lapse rate and increases convection. This generates a slight intensification in the storm. The reverse occurs during the daytime as the cloud tops absorb solar radiation. This process may be augmented by differential cooling of cloudy and clear areas.

This conceptual model is tested through the use of a numerical model. The basic model reproduces well the development of a strong hurricane from a weak tropical depression. The model storm exhibits strong, cyclonic low-level inflow and weaker anticyclonic upper-level outflow. In addition, spiral rainbands and an eye are observed during the simulation.

When the fluxes of longwave and shortwave radiation are added into the model, a definite diurnal fluctuation of intensity is evident during the early stages of the simulation. These fluctuations vary in the manner suggested by the conceptual model. This is confirmed by the oscillation of the latent heating, which peaks at night and diminishes during the day. As the storm intensifies, the fluctuations become less evident. This is to be expected, since the radiative fluxes comprise a smaller portion of the total energy budget during the later stages of the simulation.
\end{abstract}

\section{Introduction}

Research into the diurnal oscillations of tropical cyclones is a relatively recent topic of interest. In fact, only a few years have passed since Browner et al. (1977) presented their evidence for diurnal oscillations in the cirrus canopies of tropical cyclones. Using infrared satellite imagery they uncovered an apparently systematic pattern of changes in the areal extent of the cirrus canopies of tropical cyclones. These variations decreased in magnitude as the storm increased in intensity. Additionally, they speculated that 1) this oscillation is probably a manifestation of convective oscillation, 2) the decrease in magnitude may result from a better defined subsidence pattern, and 3) the oscillation in the canopy implies an oscillation in the rainfall.

More recently, Lajoie and Butterworth (1984) reported on the results of a study of the cirrus canopies of 11 tropical cyclones that occurred around Australia. In that study, a diurnal oscillation was also found, but it was approximately 12 hours out of phase with that found by Browner et al. However, because of the use of different blackbody temperatures to delineate the cirrus canopies, the two studies are not strictly comparable. Indeed, the different findings from the two studies are consistent with work by Reed (1983), which showed that in the tropics clouds with blackbody temperatures less than $218 \mathrm{~K}$ had a maximum areal extent around dawn, while the area encompassed by all clouds with blackbody temperatures less than $252 \mathrm{~K}$ reached a maximum during the afternoon.

Further support for the conclusions of Browner et al. was provided by Steranka et al. (1984). In their study of 23 Atlantic Ocean tropical cyclones that occurred during 1974-79, Fourier series analysis produced an oscillation of the cirrus canopy that was in phase with Browner et al.'s (1977) results. They also conclude that this oscillation is similar to the pattern of deep cumulus convection at small islands in the western Pacific Ocean noted by Gray and Jacobson (1977). Thus, there seems to be sufficient evidence for diurnal oscillations of the cirrus canopies produced by tropical cyclones to warrant further investigation.

If this phenomenon exists, the next problem is to attempt to discover the mechanism that produces this pattern. In this paper, the diurnal cycle of net radiation at the cloud tops is postulated as the primary cause of these oscillations. While this conceptual model is discussed in greater detail in the next section, the basic components are as follows. Radiative cooling of the cloud tops at night steepens the lapse rate and increases convection. This generates an increase in the intensity of the storm. During daylight hours, the absorption of solar radiation reduces the lapse rate, thus reducing convection. It is possible that the differential cooling of cloudy and clear areas discussed by Gray and Jacobson (1977) is also an important component of the mechanism which generates these diurnal oscillations. 
These two mechanisms can be combined in a conceptual model, as shown in the next section, and the manner in which the radiative fluxes are calculated allows for the incorporation of both phenomena into the numerical model.

This conceptual model was tested using a numerical model capable of simulating the development of tropical cyclones. The effects of the radiative fluxes were examined by performing two simulations. In the first simulation the effects of the radiative fluxes were not included in the model. The second simulation was exactly the same as the first with the exception of the inclusion of the radiative fluxes. The model and the results of these simulations are discussed in sections 3 , 4 and 5.

\section{A conceptual model of the diurnal oscillations}

The principal objective of this study was to reproduce the diurnal oscillations of tropical cyclones using a three-dimensional numerical model. However, before this was possible, it was necessary to develop a conceptual model of the processes that generate these oscillations. The model discussed below accounts for the diurnal features mentioned by Browner et al. (1977).

Browner et al. discovered apparent diurnal oscillations in the cirrus canopies of tropical cyclones while working with infrared images of North Atlantic systems produced by the SMS-1 and SMS-2 satellites during the 1974 and 1975 hurricane seasons. A fairly homogeneous sample was obtained by restricting the storms under consideration to those that 1) had cloud masses which were easily distinguishable from other nearby systems, 2) had circulations that were entirely over water, and 3 ) had been observed continuously by the satellites. The times of maximum areal extent of the cirrus canopies for these storms ranged from 1200 to 1900 LMST (Local Mean Solar Time) with an average time of occurrence of 1700 LMST. The average time of minimum cirrus coverage was 0330 LMST, but it ranged from 2330 to 0700 LMST in the individual cases. Further investigation also revealed that the amplitude of the oscillation was inversely proportional to the intensity of the storm.

The following hypothesis, which is being proposed to account for these and other manifestations of the diurnal pattern, postulates that the diurnal flux of net radiation at the cloud tops is the process responsible for generating this pattern. It is well known that tropical cyclones generally develop in regions that are initially conditionally unstable. As the low-level convergence and the corresponding vertical motion increase, the release of latent energy in the middle and upper troposphere warms these levels and produces the warm core that is characteristic of these systems. This warming also results in the lapse rate moving from conditionally unstable to a state of saturated neutrality. After this time, the hurricane maintains this condition until changes occur in the external environment.
This scenario of hurricane development ignores the potentially significant effects of radiative exchanges upon these processes. Since the release of latent energy in the middle and upper troposphere eventually produces a lapse rate of saturated neutrality, changes of stability within clouds caused by radiative exchanges of energy (Hewson, 1943) may significantly affect the buoyancy of the air. The bottoms of the clouds absorb longwave radiation from the surface while emitting nearly equal amounts of longwave radiation back to the surface. The tops of the clouds experience greater cooling at night because they emit much more longwave radiation to space than they receive as counter radiation from the gases of the upper atmosphere. This differential cooling between the top and the bottom of the tall clouds near the center of the storm causes the lapse rate to steepen and produces slightly more instability. This, in turn, results in increased convection around the core of the system.

During daytime, however, the cloud tops receive an additional input of energy in the form of shortwave radiation, and this, in combination with the release of latent energy, produces a slight warming in the upper levels. This warming reduces the lapse, rate, and thus causes more stable conditions near the center of the storm. This pattern of temperatures at the upper level (i.e., cool at night and warm during the day) is supported by measurements made by Dvorak (Browner et al., 1977) of eastern North Pacific tropical storms.

An additional mechanism that may augment these stability changes has been proposed by Gray and Jacobson (1977). They attribute the diurnal cycle of deep convection to the differences between cloudy and clear areas. Clear columns of air cool more quickly at night than cloudy areas, and this causes the air to descend. The descending air increases convergence in the lower levels of the atmosphere and produces more rising motion and precipitation in the cloudy region. This pattern is reversed during the day. Thus, this process imposes a further diurnal forcing mechanism upon the tropical cyclone.

While this may seem inconsistent with Browner et al.'s results, since the peak convection is occurring at the time of the minimum areal extent of the cirrus, it is important to realize that it will take hours for cirrus generated at the center of the storm to reach the edges of the cloud cover and increase its areal extent. Using average outflow rates, Merritt and Wexler (1967) estimate that $12-18 \mathrm{~h}$ are necessary for this process, depending on the size and intensity of the storm. Approximately the same rate of progression is given by Steranka et al. (1984). Thus, the effect of peak convection on the areal extent of a cirrus canopy would have a similar lag time.

Furthermore, by examining the effects of stability changes on rates of vertical motion, it is possible to explain the diurnal pattern of surface pressures reported by Hebert and Jarvinen (1977). Reduced vertical mo- 
tion associated with the greater stability during the daylight period means that air is not transported vertically as quickly, and thus the continued low-level convergence results in a slight rise in the surface pressures. Alternately, the increased vertical motion resulting from more unstable night conditions produces a slight decrease of the surface pressure. Lajoie and Butterworth (1984) also found a daytime oscillation with a shorter period that was superimposed on the larger diurnal oscillation. To explain this feature, they incorporate the changes in the boundary layer produced by mesoscale subsidence as proposed by Garstang (1979) into the diurnal mechanism suggested by Gray and Jacobson (1977). According to this explanation, the subsidence causes downdrafts which make the mixed layer of the boundary layer shallower, cooler and drier. This leads to a decrease in convective activity until other processes in the tropical cyclone counteract the effects of the mesoscale downdrafts. Thus, to be totally successful a simulation would have to reproduce both the diurnal and the shorter period daytime oscillations.

\section{The basic numerical model}

\section{a. The basic equations}

A three-dimensional numerical model constructed for the purpose of testing this hypothesis is discussed in this section. The criteria of separate and easily distinguishable cloud masses, circulations entirely over water, and continuous observation used by Browner et al. are easily incorporated into the model. The version of the numerical model without radiation is quite similar to a model developed by Anthes et al. (1971) and modified by Anthes (1972). In the interest of brevity, only the basic components of the model and the further modifications to Anthes' (1972) work are discussed here. The basic equations, written in the sigma coordinate system originally developed by Phillips (1957) are

$$
\begin{aligned}
\frac{\partial p^{*} u}{\partial t}=-\frac{\partial p^{*} u^{2}}{\partial x}- & \frac{\partial p^{*} u v}{\partial y}-\frac{p^{*} \partial u \dot{\sigma}}{\partial \sigma} \\
& -R T \frac{\partial p^{*}}{\partial x}-p^{*} \frac{\partial \phi}{\partial x}+p^{*} f v+F_{x} \\
\frac{\partial p^{*} v}{\partial t}=-\frac{\partial p^{*} u v}{\partial x}- & \frac{\partial p^{*} v^{2}}{\partial y}-p^{*} \frac{\partial v \dot{\sigma}}{\partial \sigma} \\
& -R T \frac{\partial p^{*}}{\partial y}-p^{*} \frac{\partial \phi}{\partial y}-p^{*} f u+F_{y} \\
\frac{\partial \phi}{\partial \sigma}= & -\frac{R T}{\sigma} \\
\frac{\partial p^{*}}{\partial t}= & -\frac{\partial p^{*} u}{\partial x}-\frac{\partial p^{*} v}{\partial y}-\frac{\partial p^{*} \dot{\sigma}}{\partial \sigma}
\end{aligned}
$$

$$
\begin{aligned}
\frac{\partial p^{*} T}{\partial t}=\frac{-\partial p^{*} u T}{\partial x}-\frac{\partial p^{*} v T}{\partial y} & -p^{*} \frac{\partial \dot{\sigma} T}{\partial \sigma} \\
& +\frac{R T \omega}{c_{p} \sigma}+\frac{p^{*}}{c_{p}} \dot{Q}+F_{k}(T) .
\end{aligned}
$$

The first two equations are the $u$-component (1) and $v$-component (2) equations of motion. This is followed by the hydrostatic equation (3), while (4) is the continuity equation and (5) is the thermodynamic equation. In these equations $x$ and $y$ are the east-west and northsouth Cartesian coordinates, and $\sigma$ is the vertical coordinate $\left(\sigma=p / p^{*}\right)$. The symbols $u$ and $v$ represent the horizontal velocity components in the $x$ and $y$ directions, respectively, and $\dot{\sigma}$ is the vertical velocity. The surface pressure is denoted by $p^{*}, \phi$ is the geopotential, $T$ is the temperature, $R$ is the specific gas constant for dry air, and $c_{p}$ is the specific heat at constant pressure. The symbols $F_{x}, F_{y}$ and $F_{k}(T)$ are subgrid-scale diffusion terms, and $Q$ is the diabatic heating term. This is the term into which the radiative exchanges are included. The $\omega$ represents the vertical velocity term in the pressure system $(\omega=d p / d t)$ and it is related to $\sigma$ by

$$
\omega=p^{*} \dot{\sigma}+\sigma \frac{d p^{*}}{d t} .
$$

Since the model explicitly accounts for transportation of water vapor, the following continuity equation for water vapor is also needed

$$
\frac{\partial p^{*} q}{\partial t}=-\frac{\partial p^{*} u q}{\partial x}-\frac{\partial p^{*} v q}{\partial y}-p^{*} \frac{\partial \dot{\sigma} q}{\partial \sigma}+S,
$$

where $q$ is the specific humidity and the $S$ term represents any source or sink of water vapor.

There are eight basic variables, $u, v, p^{*}, T, \phi, \omega, \dot{\sigma}$ and $q$ and only seven equations, so the vertical sum of (4) must be used to calculate the pressure tendency, $\partial p^{*} / \partial t$

$$
\frac{\partial p^{*}}{\partial t}=\int_{0}^{1}\left(-\frac{\partial p^{*} u}{\partial x}-\frac{\partial p^{*} v}{\partial y}-\frac{\partial p^{*} \dot{\sigma}}{\partial \sigma}\right) d \sigma
$$

\section{b. Structure of the model}

The vertical structure of the model is shown in Fig. 1. The atmosphere is divided into three layers of equal pressure thickness, representing the upper, middle and lower troposphere, and a thinner Ekman boundary layer. The decision to use four layers represents an attempt to depict more accurately the low, middle and high cloudiness associated with tropical cyclones and, in particular, the high cirrus that comprises the canopy and is so crucial to this study. The horizontal velocity components, temperature, specific humidity and vertical $p$-velocity are computed for the middle of the layer, while the geopotential and vertical velocity $(\dot{\sigma})$ are defined at the boundary of the layers. This is done to 


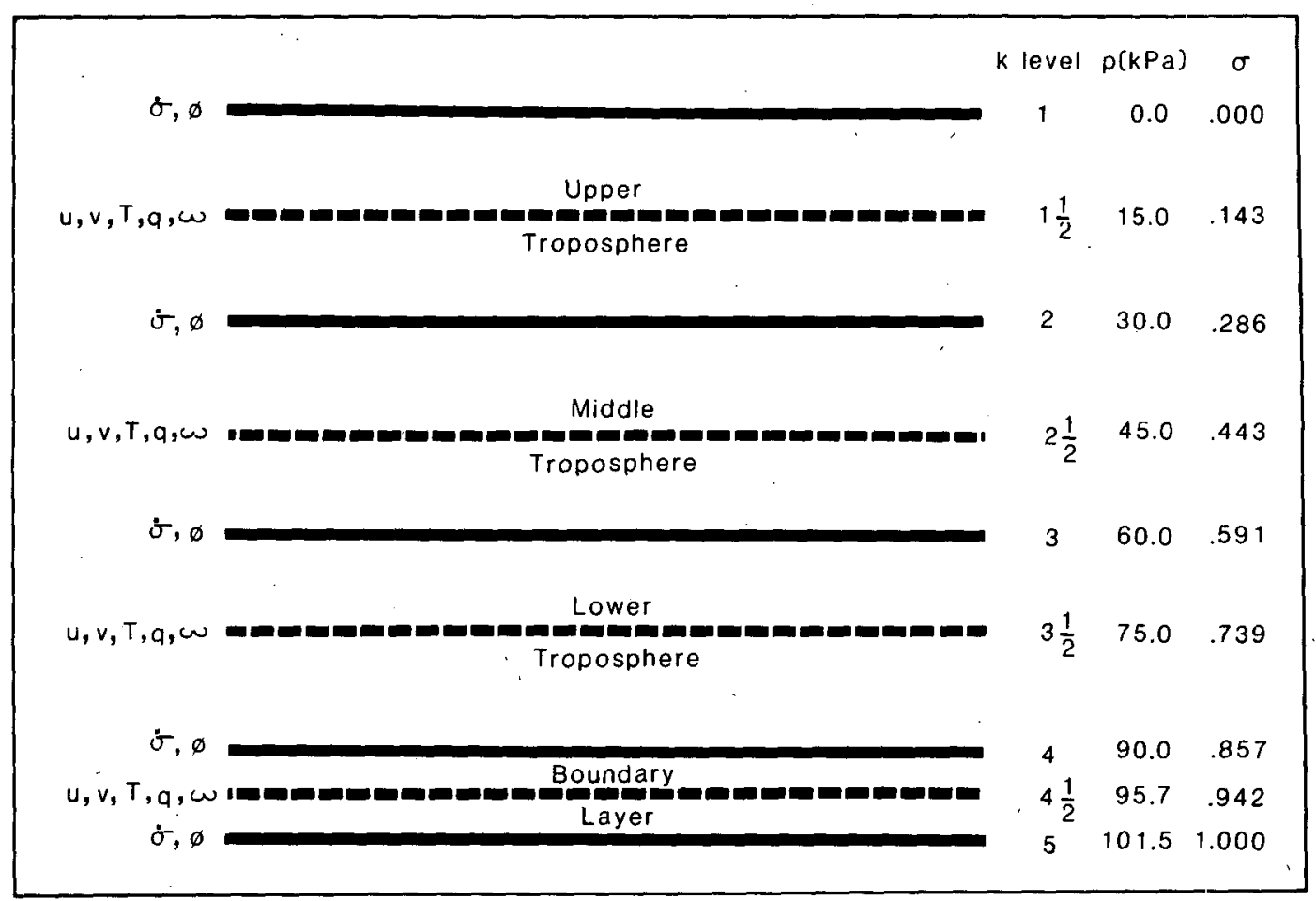

FIG. 1. Vertical staggering of variables and information levels.

maintain energy consistency in the finite difference scheme (Kurihara, 1968).

The horizontal grid is rectangular with a uniform spacing of $25 \mathrm{~km}$. This value was chosen to provide reasonable areal resolution without increasing prohibitively the computational requirements for storage and time. It is important to note that, although a reduction in grid spacing from $30 \mathrm{~km}$ used by Anthes (1972) to $25 \mathrm{~km}$ provides only a $16.7 \%$ improvement in the linear resolution, it results in a $30.5 \%$ increase in the areal resolution, which is an important consideration in a study of the areal extent of cirrus canopies. A staggered grid scheme is used in which the horizontal velocity components are calculated on the standard grid and all other variables are calculated on a grid that is offset by $45^{\circ}$. The finite difference scheme is the staggered grid S1 method described by Anthes (1972), who used this technique very successfully to improve the resolution of the pressure gradient force without a major increase in the computational requirements. The integration was performed using the method developed by Matsuno (1966) and used by Anthes et al. (1971). The time step was $37.5 \mathrm{sec}$. The lateral boundary points are approximately circular and are contained within radii of $462-487 \mathrm{~km}$.

\section{c. Subgrid-scale processes}

Even with the improved resolution of this model, it is still impossible to simulate explicitly some of the important transfers of momentum and energy that occur at smaller scales. Since many of these parameterizations are similar to those described by Anthes (1972), only the basic equations and modifications to those schemes are presented in this section. The vertical diffusion of momentum by subgrid-scale features and the fluxes of water vapor and sensible heat across the wateratmosphere boundary are treated in the manner discussed by Anthes (1972). The one modification to that method is the use of a variable drag coefficient. While Anthes (1972) chose a constant drag coefficient with a value of 0.003 , the drag coefficient in this model is specified as a linear function of wind speed. The equation used to calculate $C_{D}$ (Roll, 1965, p. 105) is given by

$$
C_{D}=0.0011+0.00004\left|V^{*}\right|
$$

where $C_{D}$ is the drag coefficient and $V^{*}$ is the velocity in the boundary layer. This is the same expression that Jones (1977) used in an asymmetric model of a tropical cyclone.

The horizontal diffusion of sensible heat and momentum is also calculated in the manner used by Anthes (1972). The equations for the lateral exchanges of horizontal momentum by subgrid-scale features in the $x$ and $y$ directions are

$$
F_{H}(u)=\frac{\partial}{\partial x}\left(K_{m} \frac{\partial p^{*} u}{\partial x}\right)+\frac{\partial}{\partial y}\left(K_{m} \frac{\partial p^{*} u}{\partial y}\right)
$$




$$
F_{H}(v)=\frac{\partial}{\partial x}\left(K_{m} \frac{\partial p^{*} v}{\partial x}\right)+\frac{\partial}{\partial y}\left(K_{m} \frac{\partial p^{*} v}{\partial y}\right)
$$

where $K_{m}$ is the eddy diffusivity for momentum, calculated from

$$
\begin{aligned}
K_{m}=3.47 & \times 10^{3} \\
+ & k^{2}(\Delta s)^{2}\left|\left[\left(\frac{\partial u}{\partial x}-\frac{\partial v}{\partial y}\right)^{2}+\left(\frac{\partial v}{\partial x}+\frac{\partial u}{\partial y}\right)^{2}\right]^{1 / 2}\right|,
\end{aligned}
$$

where $\Delta s$ is the grid spacing $(25 \mathrm{~km})$ and $k$ is von Karman's constant (0.4). It should be noted that tests conducted during preliminary experimentation showed the model to be highly sensitive to the value assigned to $K_{m}$. The model also assumes that the lateral eddy diffusivities for sensible heat transfer $\left(K_{H}\right)$ and water vapor $\left(K_{E}\right)$ are the same as $K_{m}$. Thus

$$
K_{m}=K_{H}=K_{E},
$$

and the subgrid-scale transfer of internal energy becomes

$$
F_{H}(T)=p^{*}\left[\frac{\partial}{\partial x}\left(K_{H} \frac{\partial T}{\partial x}\right)+\frac{\partial}{\partial y}\left(K_{H} \frac{\partial T}{\partial y}\right)\right] .
$$

The cumulus parameterization used in this model is described in great detail by Anthes (1972), but the additional vertical layer allows for better resolution. This method bases convection on the convergence of water vapor in the boundary layer, and convection occurs whenever the atmosphere is conditionally unstable. The vertical distribution of latent heating is designed to move the column toward a state in which the temperature and humidity are determined by the equivalent potential temperature of air at the surface, as would actually occur over a prolonged period.

In certain circumstances, supersaturation exists at some of the grid points. If this occurs in the boundary layer and the column is conditionally unstable, the excess water vapor is assumed to be condensed during the convection process in the next time step. For supersaturation in the other levels, the excess water vapor is assumed to condense as a result of large-scale uplifting and to fall as nonconvective precipitation. The latent energy, thus released, is partitioned in the remaining vertical layers in such a manner as to move the atmosphere toward saturated neutrality. For supersaturation in the lower troposphere, $45 \%$ of the condensation occurs at that level, $40 \%$ in the middle troposphere and $15 \%$ in the upper troposphere. If supersaturation occurs in the middle troposphere, $65 \%$ of the condensation takes place at that level and $35 \%$ in the upper atmosphere. All condensation resulting from supersaturation in the upper troposphere is at that level. These percentages represent the approximate proportion of water vapor that would condense in each layer if an air parcel began rising from beneath the preceding layer.

\section{The simulations \\ a. Initial conditions}

In this section, the results of two simulations performed to test the conceptual model of the diurnal oscillations introduced in section 2 are presented. The two versions of the model used to produce the results reported here differ only in the fact that, in the second simulation, exchanges of radiation are modeled explicitly, while in the first they are all set to zero. It is hoped that the comparison of these two simulations clearly demonstrates the ability of the diurnal variation of net radiation to generate a similar oscillation in the intensity of a tropical cyclone.

The initial conditions are identical for both simulations. Initially, the temperature field is only a function of sigma. With one exception, the vertical profile of temperature shown in Table 1 is taken directly from Hebert and Jordan's (1959) computation of a mean sounding for the hurricane season in the Gulf of Mexico. The one exception occurs in the upper layer ( $\sigma$ $=0.143$ ), representing the upper troposphere. At this level, the temperature was reduced from the mean sounding value of $206.6 \mathrm{~K}$ to $200.3 \mathrm{~K}$, which is a more accurate representation of the tropopause temperature. This change also produces a more realistic vertical distribution of the latent heating that occurs during the early stages of the simulation. Initially the relative humidity is $90 \%$ at all grid points.

The wind field at the start of the simulations represents an ideal vortex generated using the gradient wind balance. At the bottom of the atmosphere, the surface pressures are initialized using the following conditions:

or

$$
p^{*}=101.0-4.0 \cos \left(\frac{\pi r}{375}\right), \quad r<375 \mathrm{~km}
$$

$$
p^{*}=101.5, \quad \cdot r \geqslant 375 \mathrm{~km}
$$

where $p^{*}$ is the surface pressure in kiloPascals and $r$ is the distance from the center of the vortex in kilometers. This results in a system which has a minimum surface pressure of $107.0 \mathrm{kPa}$ and a maximum wind speed in the boundary layer of $19.4 \mathrm{~m} \mathrm{~s}^{-1}$ at a radius of 237.5 $\mathrm{km}$. The winds at the other levels are generated using the same pressure gradient and the initial temperatures defined in Table 1. Thus, the intensity of the system

TABLE 1. Initial vertical profiles of temperature and humidity.

\begin{tabular}{ccc}
\hline & $\begin{array}{c}\text { Temperature } \\
(\mathrm{K})\end{array}$ & $\begin{array}{c}\text { Relative humidity } \\
(\%)\end{array}$ \\
\hline 0.143 & 200.3 & 90 \\
0.443 & 261.6 & 90 \\
0.739 & 285.5 & 90 \\
0.942 & 293.7 & 90 \\
\hline
\end{tabular}


at the beginning of the simulation is that of a strong tropical depression.

\section{b. The basic simulation without radiation}

Before attempting to reproduce the diurnal oscillations, it is necessary to perform a basic simulation without the explicit radiative fluxes to insure that the model is capable of replicating the development of a tropical cyclone. However, since the structure of the simulated tropical cyclone is very similar to that generated by Anthes (1972) using a three-level asymmetric model, this discussion of the basic simulation is relatively brief. The initial wind field is in gradient balance and thus the radial component of the velocity is zero. In the first time step, the effects of friction are introduced and the early hours of the simulation are a period of atmospheric adjustment to this new situation. When friction is introduced, the gradient balance is destroyed and a radial component of the wind, directed inward towards the lower pressures, begins to increase in magnitude. This produces convergence, mainly in the boundary layer, which initiates convection and the warming of the upper layers of the model cyclone through the release of latent energy during condensation. Thus, while the direct effect of friction is to reduce the wind speed, the convergence resulting from this begins creating the warm core and, therefore, the intensification process. For the first $6.5 \mathrm{~h}$ of the simulation, the destructive effects exceed those favoring intensification and the maximum wind speed in the boundary layer decreases. After this time, the model storm begins a long period of intensification. This is clearly apparent in Figs. 2 and 3, which show the max- imum wind speed in the boundary layer and the minimum surface pressure plotted as functions of time.

By $92 \mathrm{~h}$ the storm effectively reaches its peak intensity and the maximum wind speeds begin to oscillate around a value of $55.5 \mathrm{~m} \mathrm{~s}^{-1}$. A similar oscillation of the minimum surface pressure also occurs around a value of $96.4 \mathrm{kPa}$. During the period of intensification, the storm maintains its generally radially symmetrical structure and exhibits many of the flow characteristics of a developing tropical cyclone. Figures 4 and 5 display typical radial profiles of radial wind speed and tangential wind speed at $72 \mathrm{~h}$. The maximum tangential speed of $50 \mathrm{~m} \mathrm{~s}^{-1}$ occurs at a distance of $37.5 \mathrm{~km}$ from the center, and the maximum inflow of $23.2 \mathrm{~m} \mathrm{~s}^{-1}$ is found just beyond this radius.

While the intensity of the model storm remains relatively constant after $92 \mathrm{~h}$, important changes are still occurring to its structure. As Fig. 6 shows, the upperlevel wind speeds at $72 \mathrm{~h}$ still exhibit a generally radial symmetry throughout the storm. While the isotachs are not concentric circles, like the pattern existing with the initially radially symmetrical vortex, the basic symmetry of the system is still evident. Twenty-four hours later, as seen in Fig. 7, the first manifestation of the spiral band in the upper outflow is evident and the asymmetrical structure of a strong tropical cyclone is more apparent. By $144 \mathrm{~h}$ the system exhibits two spiral bands in which there is strong outflow, as is shown in Fig. 8: This outflow is generated by the strong convergence in the boundary layer and the rising motion which also is now occurring in the spiral bands. As is evident in Fig. 10, the northern spiral band is the stronger of the two. After developing, these spiral bands rotate cyclonically around the center of the storm and

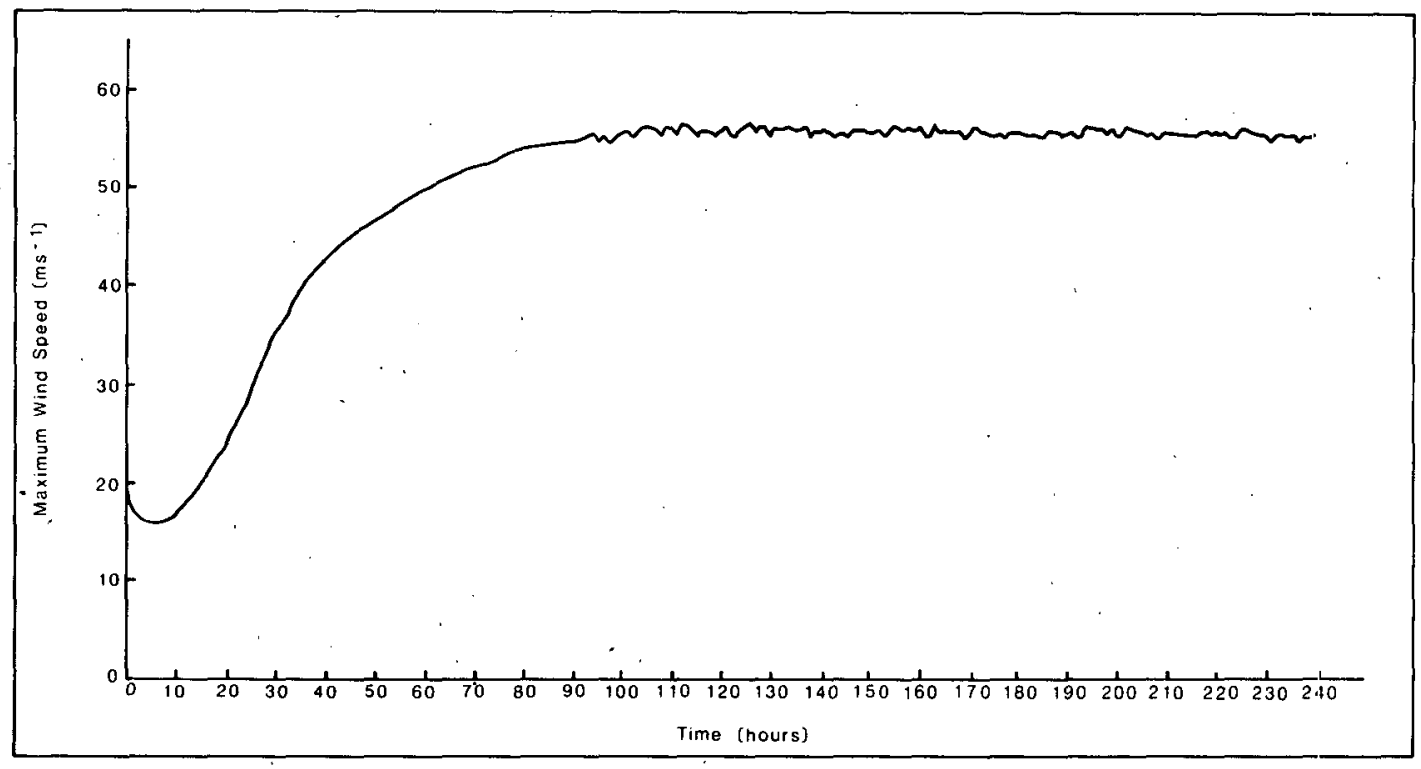

FIG. 2. Maximum boundary layer wind speed vs time for nonradiative simulation. 


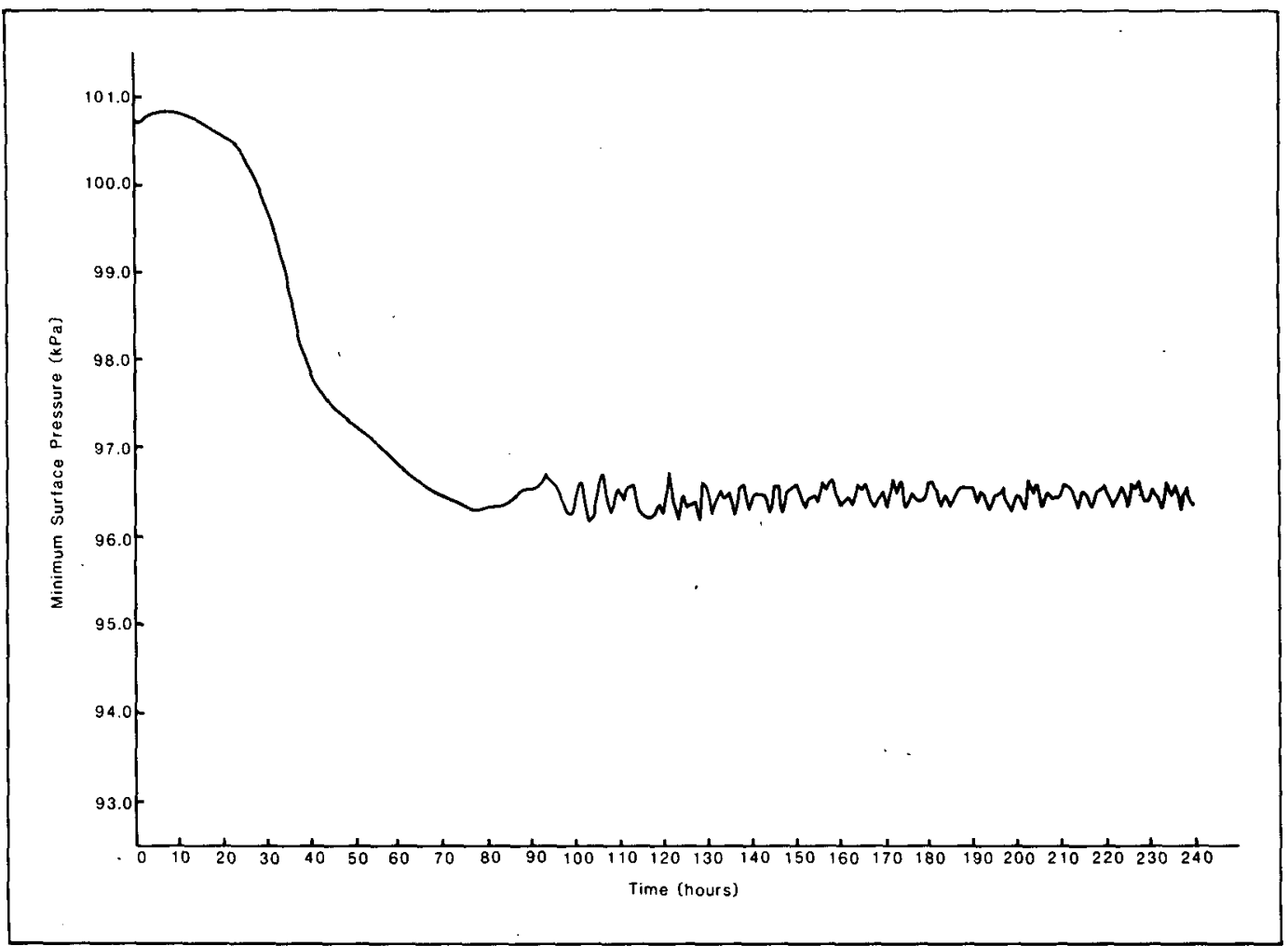

FIG. 3. As in Fig. 2 but for minimum surface pressure.

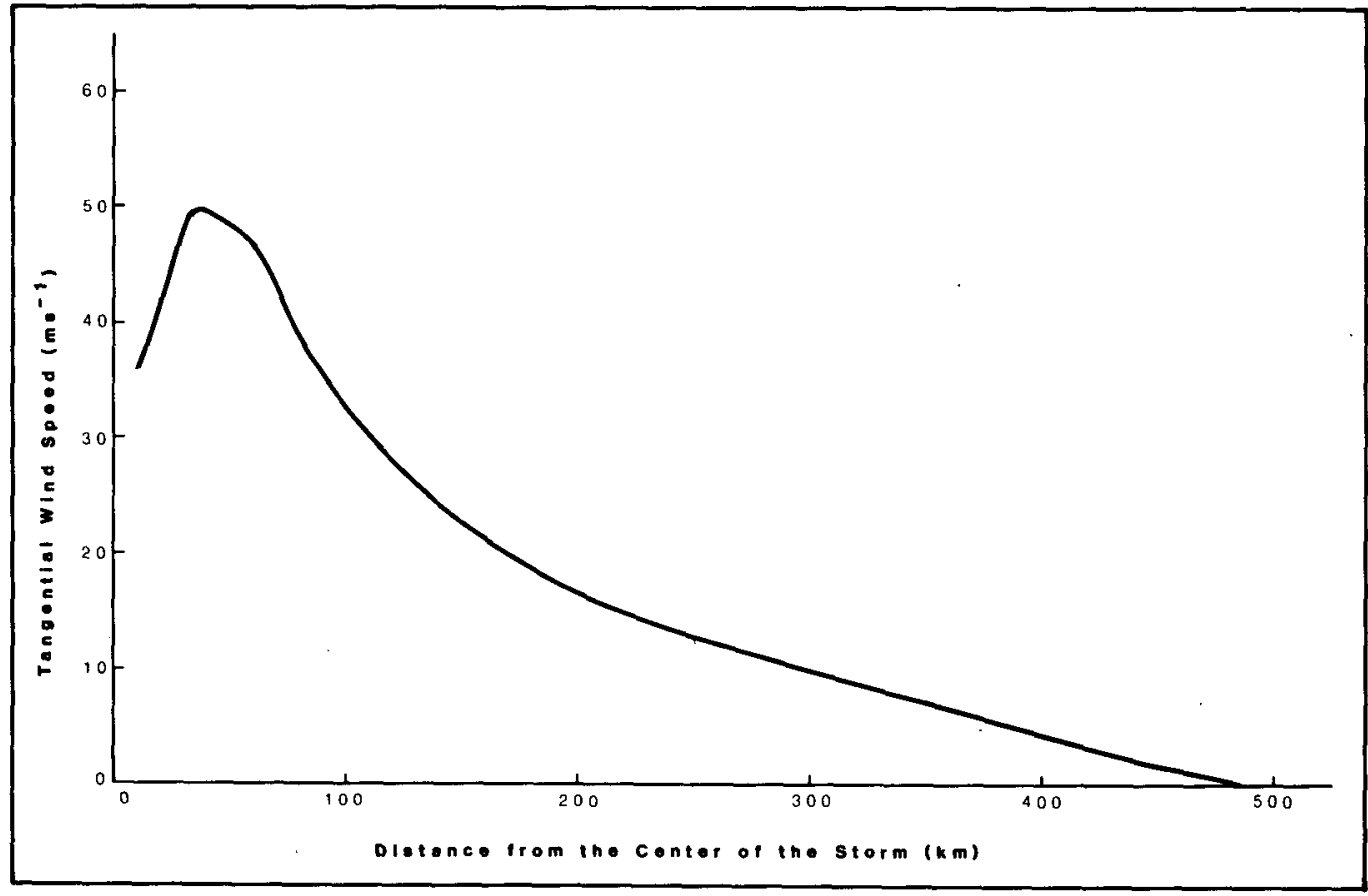

FIG. 4. Radial profile of radial component of boundary layer wind at $72 \mathrm{~h}$ for nonradiative simulation. 


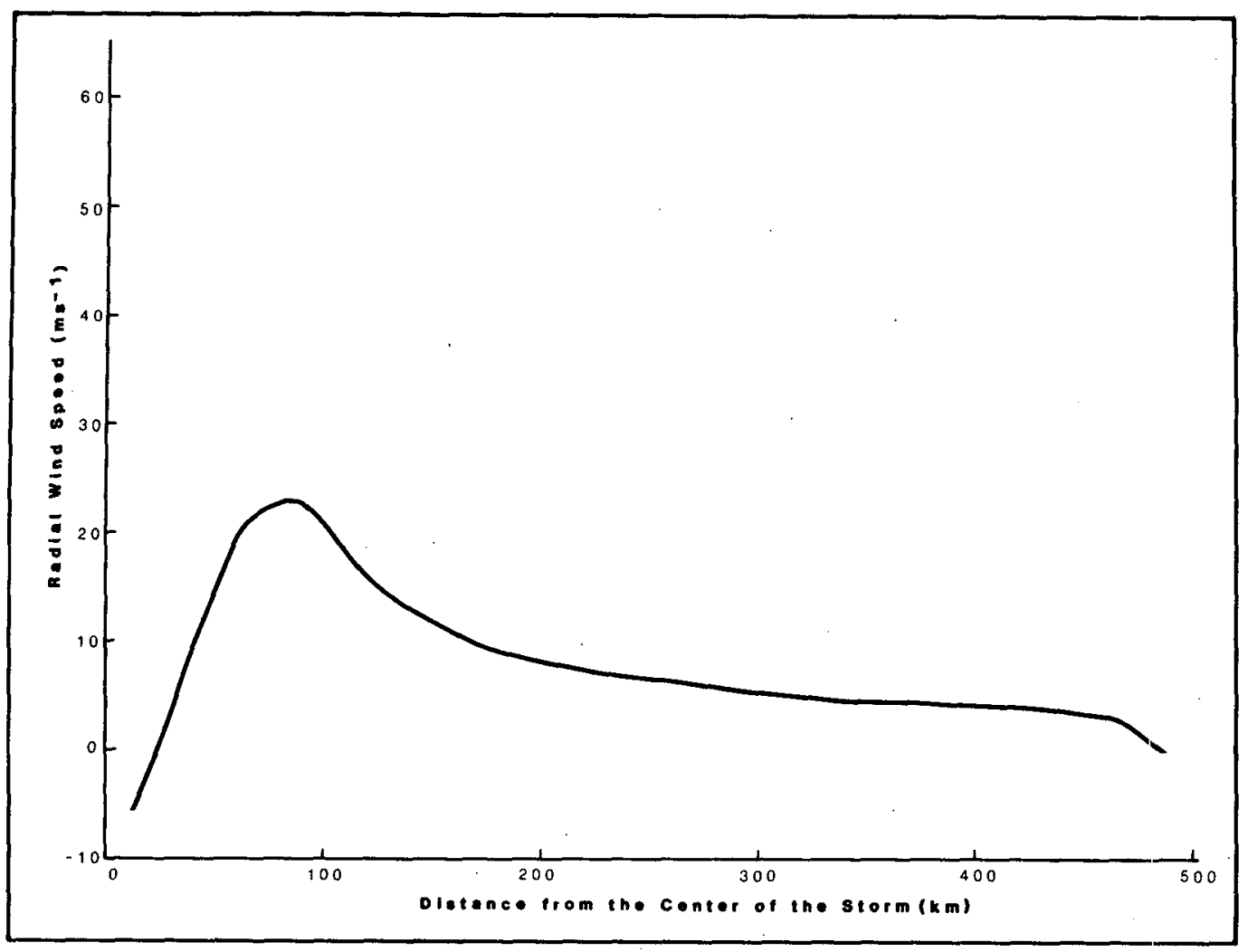

FIG. 5. As in Fig. 4 but of the tangential component.

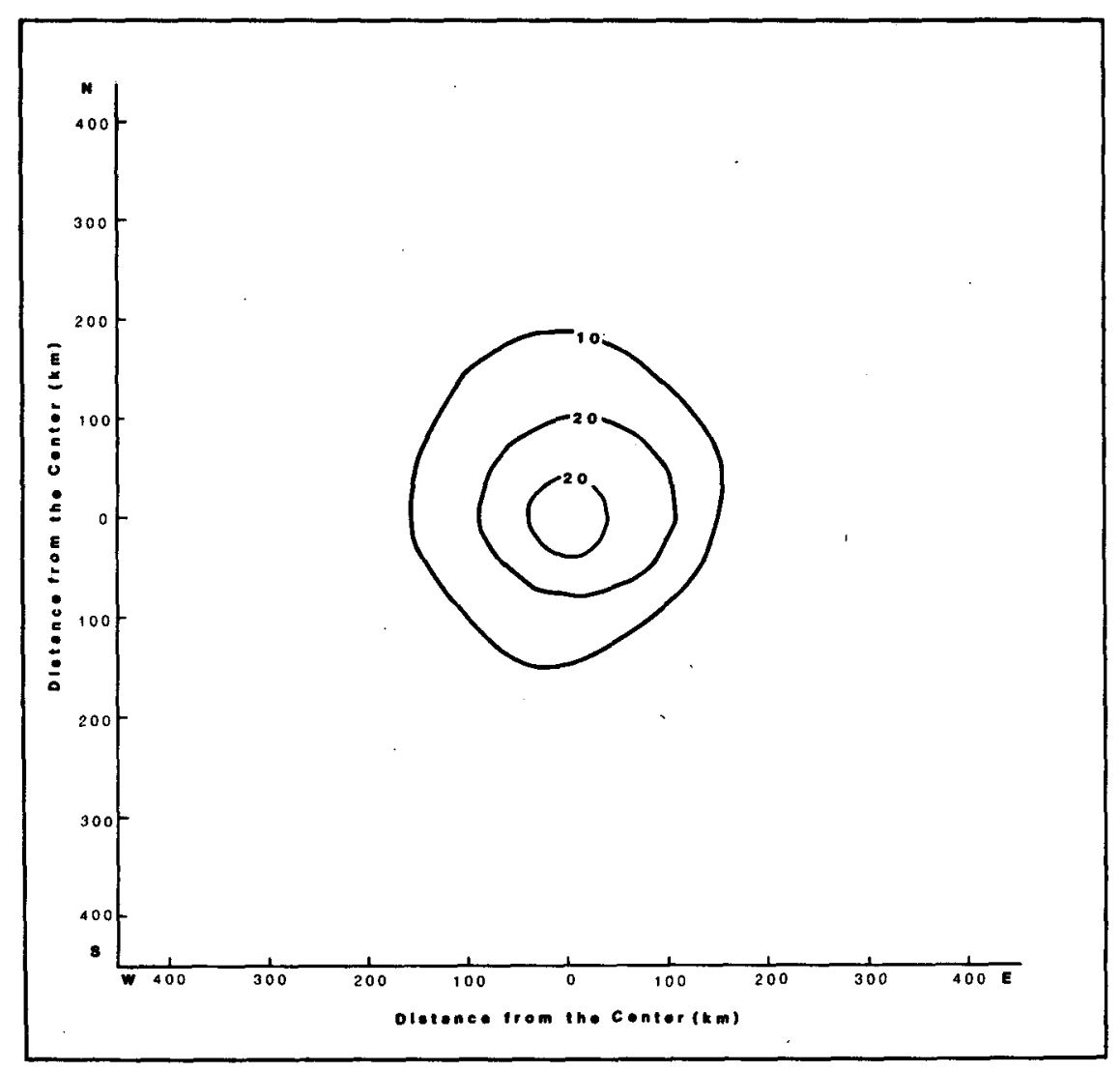

Fig. 6. Isotach analysis $\left(\mathrm{m} \mathrm{s}^{-1}\right)$ of the upper troposphere at $72 \mathrm{~h}$ for nonradiative simulation. 


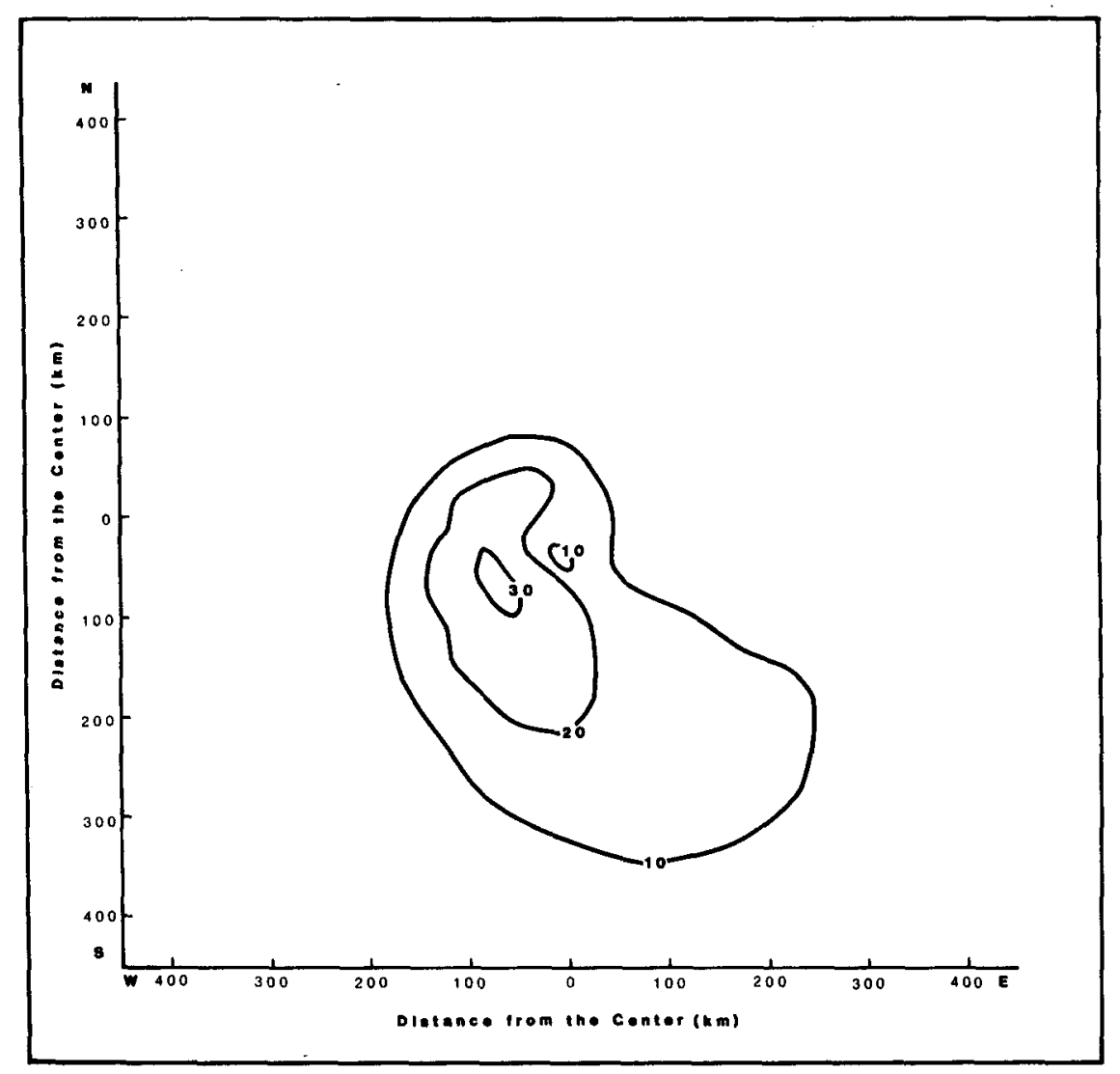

FIG. 7. As in Fig. 6 but at $96 \mathrm{~h}$.

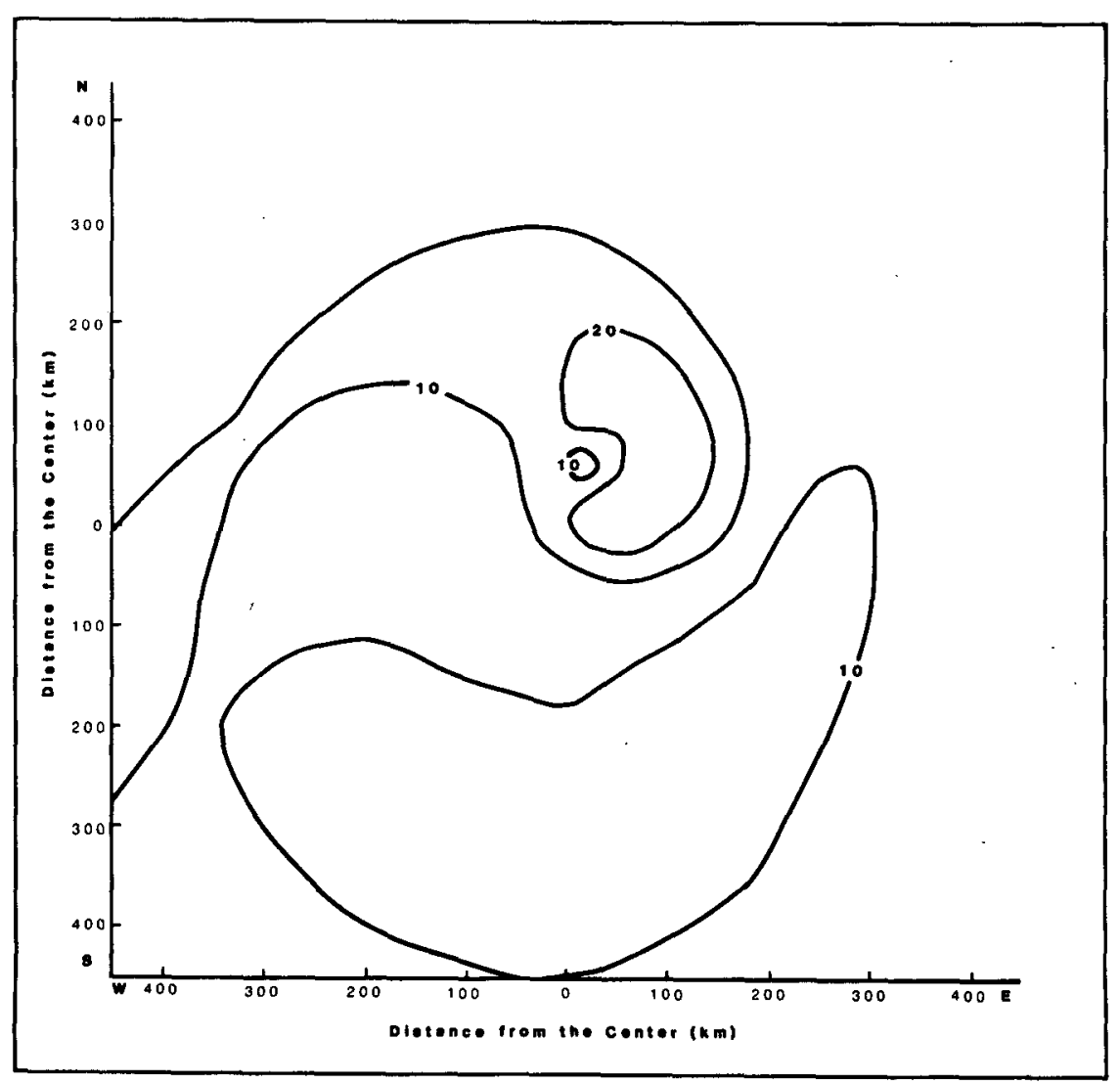

FIG. 8. As in Fig. 6 but at 144 h. 
slowly move outwards. This is similar to Anthes' (1972) findings using a three-level asymmetric model.

\section{c. Inclusion of the radiative fluxes}

Since the results presented in the previous section establish that the basic model accurately reproduces the important features of a tropical cyclone, the next step is to incorporate the radiative fluxes explicitly into the model before attempting to simulate the diurnal oscillations of the tropical cyclone. Both solar (shortwave) and terrestrial (longwave) radiative fluxes are present in the expanded version of the model. In addition, the method of computing the net radiation contains the effects of the clouds and the important atmospheric gases.

Before describing this computational scheme in detail, it is necessary to distinguish between an information level at which information on temperature, moisture and clouds is available and the layers separating these levels. No data on temperature, moisture or cloud are available for these intervening thicknesses. The effects of clouds are assumed to occur within infinitesimally small layers which are centered on the information levels. Thus absorption, emission and reflection by clouds are calculated for the levels. Between these are layers of finite sigma thicknesses and it is assumed that all absorption, scattering and emission by atmospheric gases occur within these layers. This vertical structure is shown in Fig. 9. Level 0 represents the top of the atmosphere, while levels 1,2 and 3 correspond to the upper, middle and lower troposphere, respectively. Because no clouds exist in level 4 , it is included as part of layer 4 , and level 5 represents the surface.

The effects of the radiative fluxes on the temperature are incorporated into the diabatic heating term, $\dot{Q}$,

$$
\dot{Q}_{\mathrm{RAD}}=\left(\frac{d T}{d t}\right)_{\mathrm{RAD}}=\frac{1}{\rho c_{p}} \frac{d Q^{*}}{d t z}
$$

where $\dot{Q}$ is the net radiation and all other symbols have their standard meanings (Paltridge and Platt, 1976). Changing the vertical gradient to sigma coordinates results in

$$
\dot{Q}_{\mathrm{RAD}}=-\frac{g}{p^{*} c_{p}} \frac{d Q^{*}}{d \sigma} .
$$

It is possible to separate this into the longwave and shortwave fluxes. Thus

$$
\dot{Q}_{\mathrm{RAD}}=-\frac{g}{p^{*} c_{p}}\left[\frac{d L^{*}}{d \sigma}+\frac{d K^{\mathrm{*}}}{d \sigma}-\right]
$$

where $L^{*}$ is the net longwave radiation and $K^{*}$ is the net shortwave radiation. In order to simplify this discussion, the method for handling the longwave fluxes is described first and then the scheme for the shortwave fluxes is presented.

The vertical gradient of net longwave radiation for level 1 is given by

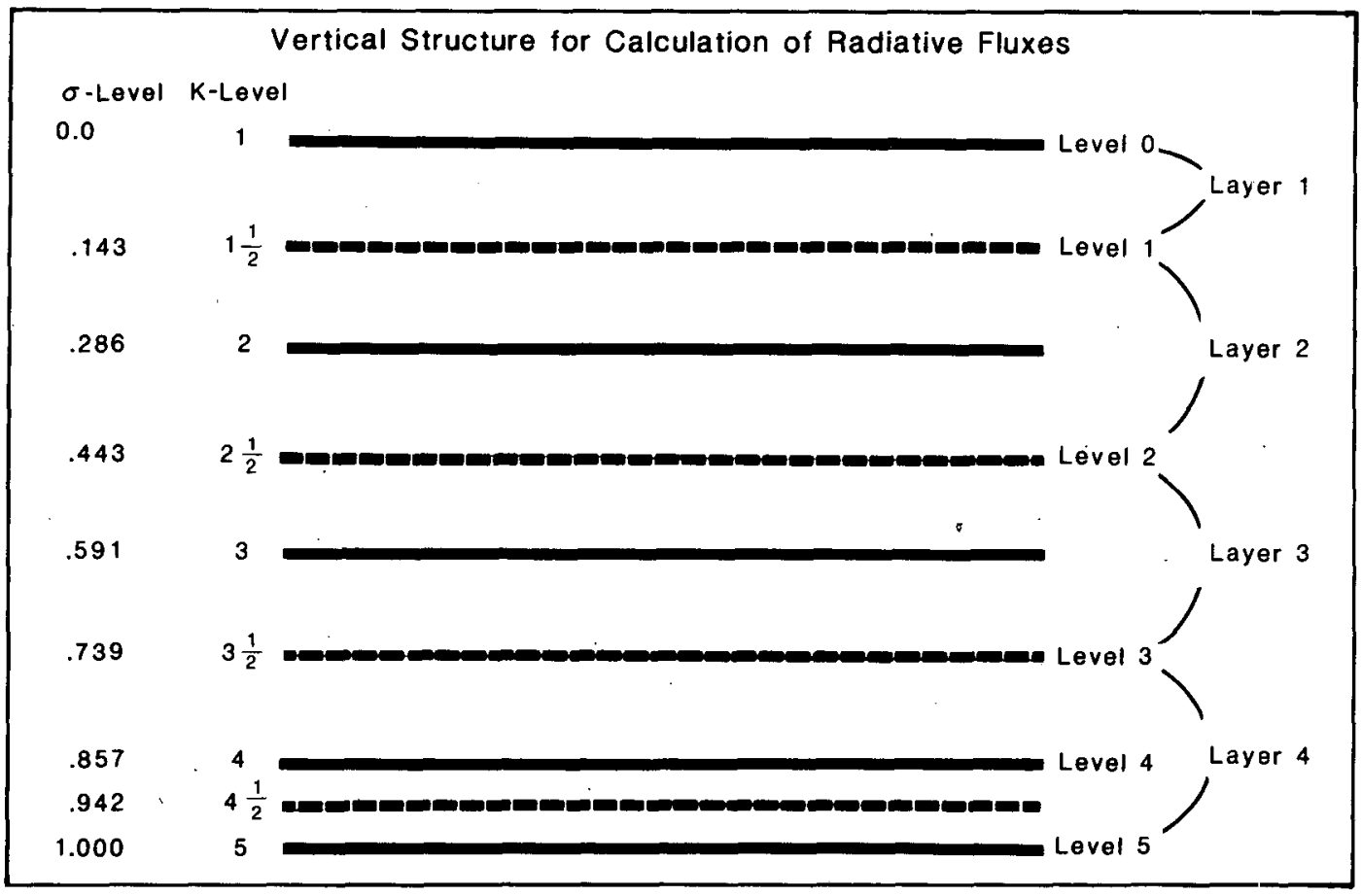

FIG. 9. Vertical structure used for calculation of the radiative fluxes. 


$$
\left(\frac{d L^{*}}{d \sigma}\right)_{1}=\frac{L_{0}^{*}-L_{2}^{*}}{\sigma_{0}-\sigma_{2}}
$$

where the subscripts refer to the information levels.

Expanding the net longwave terms gives

$$
\left(\frac{d L^{*}}{d \sigma}\right)_{1}=\frac{L \downarrow_{0}-L \uparrow_{0}-L \downarrow_{2}+L \uparrow_{2}}{\sigma_{0}-\sigma_{2}} .
$$

All incoming or outgoing fluxes are calculated for the side of the information level closest to the level for which the temperature tendency is desired. An example of this for level 1 is seen in Fig. 10. For simplicity it is assumed that insignificant amounts of longwave radiation enter the top of the model. Thus,

$$
L \downarrow_{0}=0 \text {. }
$$

The equations for the other three fluxes in (71) are

$$
\begin{aligned}
& L \uparrow_{0}=\epsilon_{(1)} \sigma\left(\frac{T_{0}+T_{1}}{2}\right)^{4}+\epsilon_{1} \sigma T_{1}^{4}\left(1-\epsilon_{(1)}\right)+\epsilon_{(2)} \sigma\left(\frac{T_{1}+T_{2}}{2}\right)^{4} \\
& \times\left(1-\epsilon_{1}\right)\left(1-\epsilon_{(1)}\right)+\epsilon_{2} \sigma T_{2}^{4}\left(1-\epsilon_{(2)}\right)\left(1-\epsilon_{1}\right)\left(1-\epsilon_{(1)}\right) \\
& +\epsilon_{(3)} \sigma\left(\frac{T_{2}+T_{3}}{2}\right)^{4}\left(1-\epsilon_{2}\right)\left(1-\epsilon_{(2)}\right)\left(1-\epsilon_{1}\right)\left(1-\epsilon_{(1)}\right) \\
& +\epsilon_{3} \sigma T_{3}^{4}\left(1-\epsilon_{(3)}\right)\left(1-\epsilon_{2}\right)\left(1-\epsilon_{(2)}\right)\left(1-\epsilon_{1}\right)\left(1-\epsilon_{(1)}\right) \\
& +\epsilon_{(4)} \sigma\left(\frac{T_{3}+T_{4}+T_{5}}{3}\right)^{4}\left(1-\epsilon_{3}\right)\left(1-\epsilon_{(3)}\right)\left(1-\epsilon_{2}\right)\left(1-\epsilon_{(2)}\right) \\
& \times\left(1-\epsilon_{1}\right)\left(1-\epsilon_{(1)}\right)+\epsilon_{5} \sigma T_{5}^{4}\left(1-\epsilon_{(4)}\right)\left(1-\epsilon_{3}\right)\left(1-\epsilon_{(3)}\right) \\
& \quad \times\left(1-\epsilon_{2}\right)\left(1-\epsilon_{(2)}\right)\left(1-\epsilon_{1}\right)\left(1-\epsilon_{(1)}\right), \quad(21) \\
& L \downarrow_{2}=\epsilon_{(2)} \sigma\left(\frac{T_{1}+T_{2}}{2}\right)^{4}+\epsilon_{1} \sigma T_{1}^{4}\left(1-\epsilon_{(2)}\right) \\
& +\epsilon_{(1)} \sigma\left(\frac{T_{0}+T_{1}}{2}\right)^{4}\left(1-\epsilon_{1}\right)\left(1-\epsilon_{(2)}\right), \quad(22)
\end{aligned}
$$

and

$$
\begin{aligned}
L \uparrow_{2}= & \epsilon_{2} \sigma T_{2}{ }^{4}+\epsilon_{(3)} \sigma\left(\frac{T_{2}+T_{3}}{2}\right)^{4}\left(1-\epsilon_{2}\right) \\
& +\epsilon_{3} \sigma T_{3}{ }^{4}\left(1-\epsilon_{(3)}\right)\left(1-\epsilon_{2}\right) \\
& +\epsilon_{(4)} \sigma\left(\frac{T_{3}+T_{4}+T_{5}}{3}\right)^{4}\left(1-\epsilon_{3}\right)\left(1-\epsilon_{(3)}\right)\left(1-\epsilon_{2}\right) \\
& +\epsilon_{5} \sigma T_{5}{ }^{4}\left(1-\epsilon_{(4)}\right)\left(1-\epsilon_{3}\right)\left(1-\epsilon_{(3)}\right)\left(1-\epsilon_{2}\right),
\end{aligned}
$$

where $\sigma$ is the Stefan-Boltzmann constant and $\epsilon$ is the emissivity. The subscripts within parentheses refer to layers and those without to information levels. Since no temperatures are known for the layers, an average value is calculated using the values from the information levels on the edges of the layers. In layer 4 an additional temperature, from the middle of the boundary layer (level 4), is included to give a more representative average.
Similar sets of equations are used to calculate the net longwave effects on levels 2 and 3 . For level 2 these are

$$
\left(\frac{d L^{*}}{d \sigma}\right)_{2}=\frac{L \downarrow_{1}-L \uparrow_{1}-L \downarrow_{3}+L \uparrow_{3}}{\sigma_{1}-\sigma_{3}},
$$

where

$$
L \uparrow_{1}=\epsilon_{1} \sigma T_{1}^{4}+\epsilon_{(1)} \sigma\left(\frac{T_{0}+T_{1}}{2}\right)^{4}\left(1-\epsilon_{1}\right),
$$

$$
\begin{aligned}
& L \downarrow_{1}=\epsilon_{(2)} \sigma\left(\frac{T_{1}+T_{2}}{2}\right)^{4}+\epsilon_{2} \sigma T_{2}{ }^{4}\left(1-\epsilon_{(2)}\right) \\
& +\epsilon_{(3)} \sigma\left(\frac{T_{2}+T_{3}}{2}\right)^{4}\left(1-\epsilon_{2}\right)\left(1-\epsilon_{(2)}\right) \\
& +\epsilon_{3} \sigma T_{3}^{4}\left(1-\epsilon_{(3)}\right)\left(1-\epsilon_{2}\right)\left(1-\epsilon_{(2)}\right) \\
& +\epsilon_{(4)} \sigma\left(\frac{T_{3}+T_{4}+T_{5}}{3}\right)^{4}\left(1-\epsilon_{3}\right)\left(1-\epsilon_{(3)}\right)\left(1-\epsilon_{2}\right)\left(1-\epsilon_{(2)}\right) \\
& +\epsilon_{5} \sigma T_{5}{ }^{4}\left(1-\epsilon_{(4)}\right)\left(1-\epsilon_{3}\right)\left(1-\epsilon_{(3)}\right)\left(1-\epsilon_{2}\right)\left(1-\epsilon_{(2)}\right)(26) \\
& L \downarrow_{3}=\epsilon_{(3)} \sigma\left(\frac{T_{2}+T_{3}}{2}\right)^{4}+\epsilon^{4} \sigma T_{2}{ }^{4}\left(1-\epsilon_{(3)}\right)+\epsilon_{(2)} \sigma\left(\frac{T_{1}+T_{2}}{2}\right)^{4} . \\
& \quad \times\left(1-\epsilon_{2}\right)\left(1-\epsilon_{(3)}\right)+\epsilon_{1} \sigma T_{1}{ }^{4}\left(1-\epsilon_{(2)}\right)\left(1-\epsilon_{2}\right)\left(1-\epsilon_{(3)}\right) \\
& +\epsilon_{(1)} \sigma\left(\frac{T_{0}+T_{1}}{2}\right)^{4}\left(1-\epsilon_{1}\right)\left(1-\epsilon_{(2)}\right)\left(1-\epsilon_{2}\right)\left(1-\epsilon_{(3)}\right),(27) \\
& L \uparrow_{3}=\epsilon_{3} \sigma T_{3}{ }^{4}+\epsilon_{(4)} \sigma\left(\frac{T_{3}+T_{4}+T_{5}}{3}\right)^{4}\left(1-\epsilon_{3}\right)
\end{aligned}
$$

Similarly for level 3

$$
\left(\frac{d L^{*}}{d \sigma}\right)_{3}=\frac{L \downarrow_{2}-L \uparrow_{2}-L \downarrow_{5}+L \uparrow_{5}}{\sigma_{2}-\sigma_{5}},
$$

where

$$
\begin{aligned}
L \downarrow_{2}= & \epsilon_{2} \sigma T_{2}{ }^{4}+\epsilon_{(2)} \sigma\left(\frac{T_{2}+T_{1}}{2}\right)^{4}\left(1-\epsilon_{2}\right) \\
& +\epsilon_{1} \sigma T_{1}^{4}\left(1-\epsilon_{(2)}\right)\left(1-\epsilon_{2}\right)+\epsilon_{(1)} \sigma\left(\frac{T_{0}+T_{1}}{2}\right)^{4} \\
& \times\left(1-\epsilon_{1}\right)\left(1-\epsilon_{(2)}\right)\left(1-\epsilon_{2}\right), \\
L \uparrow_{2}= & \epsilon_{(3)} \sigma\left(\frac{T_{2}+T_{3}}{2}\right)^{4}+\epsilon_{3} \sigma T_{3}{ }^{4}\left(1-\epsilon_{(3)}\right) \\
& +\epsilon_{(4)} \sigma\left(\frac{T_{3}+T_{4}+T_{5}}{3}\right)^{4}\left(1-\epsilon_{3}\right)\left(1-\epsilon_{(3)}\right) \\
& +\epsilon_{5} \sigma T_{5}^{4}\left(1-\epsilon_{(4)}\right)\left(1-\epsilon_{3}\right)\left(1-\epsilon_{(3)}\right), \\
L \downarrow_{5}= & \epsilon_{(4)} \sigma\left(\frac{T_{3}+T_{4}+T_{5}}{3}\right)^{4}+\epsilon_{3} \sigma T_{3}^{4}\left(1-\epsilon_{(4)}\right) \\
& +\epsilon_{(3)} \sigma\left(\frac{T_{2}+T_{3}}{2}\right)^{4}\left(1-\epsilon_{3}\right)\left(1-\epsilon_{(4)}\right)
\end{aligned}
$$




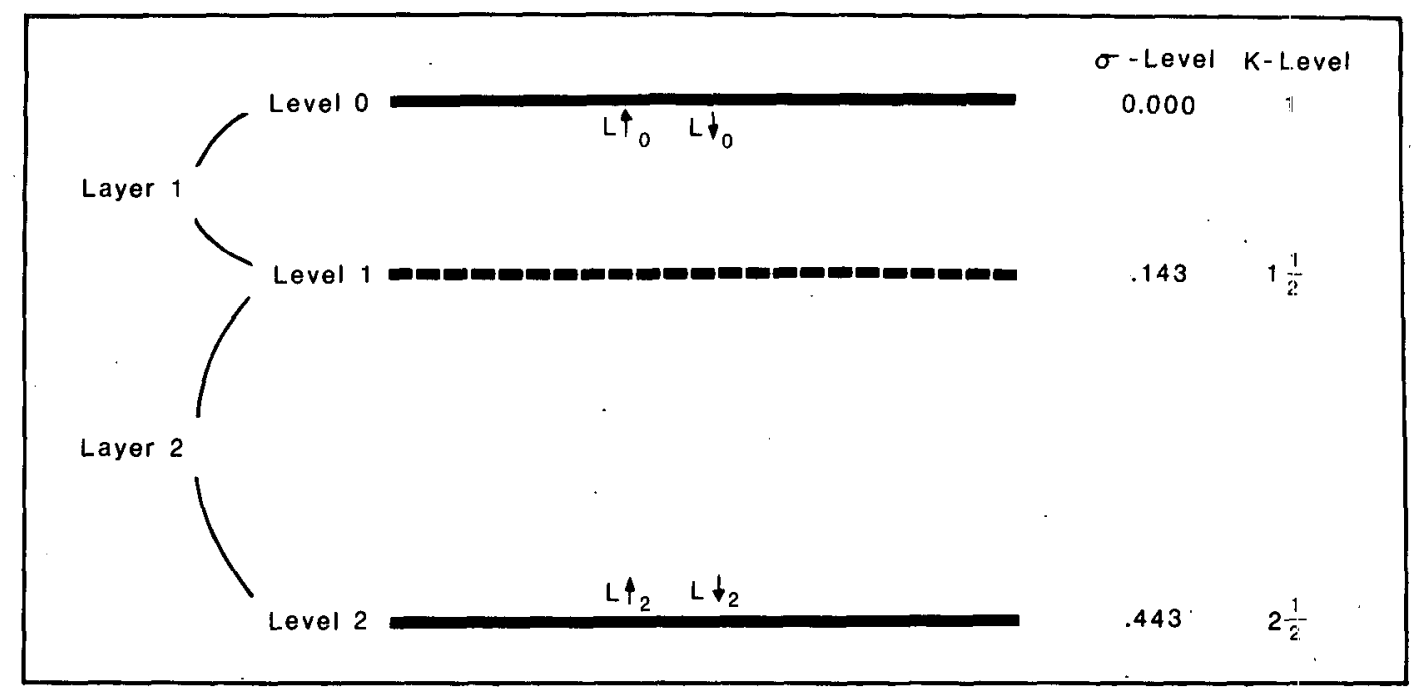

FIG. 10. Locations of incoming and outgoing longwave fluxes in calculations of the net longwave heating of level 1 .

$$
\begin{aligned}
& +\epsilon_{2} \sigma T_{2}^{4}\left(1-\epsilon_{(3)}\right)\left(1-\epsilon_{3}\right)\left(1-\epsilon_{(4)}\right) \\
& +\epsilon_{(2)} \sigma\left(\frac{T_{1}+T_{2}}{2}\right)^{4}\left(1-\epsilon_{2}\right)\left(1-\epsilon_{(3)}\right)\left(1-\epsilon_{3}\right)\left(1-\epsilon_{(4)}\right) \\
& +\epsilon_{1} \sigma T_{1}{ }^{4}\left(1-\epsilon_{(2)}\right)\left(1-\epsilon_{2}\right)\left(1-\epsilon_{(3)}\right)\left(1-\epsilon_{3}\right)\left(1-\epsilon_{(4)}\right) \\
& +\epsilon_{(1)} \sigma\left(\frac{T_{0}+T_{1}}{2}\right)^{4}\left(1-\epsilon_{1}\right)\left(1-\epsilon_{(2)}\right) \\
& \quad \times\left(1-\epsilon_{2}\right)\left(1-\epsilon_{(3)}\right)\left(1-\epsilon_{3}\right)\left(1-\epsilon_{(4)}\right), \\
& L \uparrow_{5}=\epsilon_{5} \sigma T_{5}^{4} .
\end{aligned}
$$

No radiative effects are calculated for levels 4 or 5 . In the case of level 4 , this is the middle of the boundary layer and no clouds exist below this level. Thus the clear air below this level is simply included in layer 4 . Level 5 represents the sea surface. To avoid possible effects due to changes in sea surface temperatures, it is assumed that the upper ocean is well mixed and the temperature is held constant. This makes it unnecessary to calculate the radiative effects on the surface temperatures.

To complete the treatment of the longwave radiation it is necessary to determine the emissivities of the layers and the levels. Since the effects of the atmospheric gases are represented by the layers, their longwave emissivities are calculated as functions of the water vapor, carbon dioxide and ozone concentrations in the layer. The emissivity of a layer is determined through the use of an approximation described by Paltridge and Platt (1976) and is given by

$$
\epsilon_{(i)}=\epsilon_{(i)}^{\mathrm{O}_{3}}+\epsilon_{(i)}^{\mathrm{CO}_{2}}+\epsilon_{(i)}^{\mathrm{H}_{2} \mathrm{O}}-\Delta \epsilon_{(i)}^{\mathrm{H}_{2} \mathrm{O}-\mathrm{CO}_{2} \text { overlap. }}
$$

Thus, the layer emissivity is the sum of the individual emissivities of ozone, carbon dioxide and water vapor minus an amount subtracted because of the overlap- ping emission spectra of water vapor and carbon dioxide.

Examination of each of these gases individually provides the easiest way of separating their radiative effects. Ozone is the simplest to treat because it is assumed that it is only a significant contributor to the emissivity of layer 1 . Hence,

$$
\epsilon_{(2)}^{O_{3}}=\epsilon_{(3)}^{O_{3}}=\epsilon_{(4)}^{O_{3}}=0 \text {. }
$$

The depth of ozone in the atmosphere under normal temperature and pressure given by Hitchfeld and Houghton (1961) is $2.3 \mathrm{~mm}$. This is then corrected for pressure using

$$
h_{\mathrm{O}_{3}}^{\prime}=h_{\mathrm{O}_{3}} p m_{\mathrm{O}_{3}} / R^{*} T,
$$

where $h_{\mathrm{O}_{3}}^{\prime}$ is the optical depth of ozone, $h_{\mathrm{O}_{3}}$ is the depth of ozone and $m_{\mathrm{O}_{3}}$ is the molecular weight of ozone. After modifying $h_{\mathrm{O}_{3}}^{\prime}$ so that it is in units of $\mathrm{g} \mathrm{cm}^{-2}$, the table of Staley and Jurica (1970) is used to find the

\begin{tabular}{|c|c|c|c|c|c|}
\hline \multirow[b]{2}{*}{ Layer } & \multirow[b]{2}{*}{$\epsilon_{(i)^{3}}^{o_{3}}$} & \multirow[b]{2}{*}{$\epsilon_{(i)}^{\mathrm{CO}_{2}}$} & \multirow[b]{2}{*}{$\epsilon_{(i)}^{\mathrm{H}} 2 \mathrm{O}$} & \multicolumn{2}{|c|}{$\mathrm{H}_{2} \mathrm{O}-\mathrm{CO}_{2}$ overlap } \\
\hline & & & & $\Delta \epsilon_{(\mathbf{i})}$ & $\epsilon_{(i)}$ \\
\hline 1 & 0.001 & 0.007 & 0.000 & 0.000 & 0.008 \\
\hline 2 & 0.000 & 0.020 & 0.456 & 0.001 & 0.474 \\
\hline 3 & 0.000 & 0.028 & 0.623 & 0.012 & 0.638 \\
\hline 4 & 0.000 & 0.031 & 0.776 & 0.025 & 0.783 \\
\hline
\end{tabular}
emissivity of ozone in layer 1 . This is given with the other terms in (36) in Table 2. (Note that due to rounding, the layer emissivities may not exactly equal the sum of the individual components.) In calculating the portion of the emissivity due to carbon dioxide, the constant mixing ratio of $0.456 \times 10^{-3}$ of Wetherald and Manabe (1980) is used. This is adjusted using

TABLE 2. Layer emissivities for longwave radiation. 


$$
h_{\mathrm{CO}_{2}}=-\frac{q_{\mathrm{CO}_{2}}}{g p_{0}} \int_{P_{\text {botrom }}}^{P_{\mathrm{top}}} p d p
$$

where $h_{\mathrm{CO}_{2}}$ is the optical depth of carbon dioxide for the layer and $q_{\mathrm{CO}_{2}}$ is the mixing ratio of carbon dioxide. The table of Staley and Jurica (1970) for carbon dioxide is then used to derive the emissivities presented in Table 2.

The portion of the layer emissivity due to water vapor is conceptually the most difficult of the three gases. It is known that there are relatively warm dry areas in a tropical cyclone (e.g., the eye) and saturated areas in the tall clouds surrounding the eye. This problem is somewhat ameliorated by the fact that the relative humidity is initially $90 \%$ at all points in the model. This tends to reduce the range of relative humidity so that the largest changes occur in the upper layer where there is very little water vapor. To reduce further the complexity of this problem, an average profile of mixing ratio is used. This profile results from taking the mean of the range of mixing ratios produced by the model during the basic simulation. While far from perfect, this approach represents a compromise between more complicated schemes that require larger amounts of computer resources and simpler methods that insufficiently reproduce the effects of water vapor. The mean profile used for the radiative simulation is presented in Table 3.

The mean mixing ratio for a layer $\bar{q}$ is used to calculate the optical depth of the water vapor, $u$, by

$$
u=-\frac{\bar{q}}{g P_{0}} \int_{P_{\text {bortom }}}^{P_{\text {top }}} p d p \text {. }
$$

This value is then used to determine the emissivity of the water vapor and the water vapor-carbon dioxide overlap given in Staley and Jurica's (1970) tables. These values are also presented in Table 2 . The emissivity for water vapor in layer 1 is assumed to be zero because it is assumed that the amount of water vapor in this layer (i.e., above the tropopause) makes it an insignificant emission source.

The longwave emissivities of levels 1-3 represent those for high, middle and low clouds, respectively. If no clouds are present, the emissivity is zero. If nonconvective clouds are present, then the emissivities are specified using the values taken from Paltridge and Platt

TABLE 3. Mean values of mixing ratio used to calculate the portion of the longwave emissivity due to water vapor.

\begin{tabular}{cc}
\hline Level & Mean mixing ratio \\
\hline 1 & $1.4346 \times 10^{-5}$ \\
2 & $3.3613 \times 10^{-3}$ \\
3 & $1.0872 \times 10^{-2}$ \\
4 & $1.8697 \times 10^{-2}$ \\
5 & $2.527 \times 10^{-2}$ \\
\hline
\end{tabular}

(1976). Thus, $\epsilon_{1}=0.3, \epsilon_{2}=0.9$ and $\epsilon_{3}=1.0$. If the cloud parameterization produces a deep convective cloud, it is assumed that all significant emission or absorption occurs at the edges of the cloud. Thus, $\epsilon_{1}$ $=\epsilon_{(2)}=\epsilon_{2}=\epsilon_{(3)}=\epsilon_{3}=1.0$. The emissivity of the sea surface is assumed to be 1.0 .

Shortwave radiation is treated using the same vertical organization of layers and levels discussed for longwave radiation. Thus, for level 1

$$
\left(\frac{d K^{*}}{d \sigma}\right)_{1}=\frac{K \downarrow_{0}-K \uparrow_{0}-K \downarrow_{2}+K \uparrow_{2}}{\sigma_{0}-\sigma_{2}}
$$

where

$$
K \downarrow_{0}=K_{0}=I_{0} \cos z
$$

$$
\begin{gathered}
K \uparrow_{0}=\frac{1}{2} \gamma_{(1)} K_{0}+\alpha_{1} t_{(1)}{ }^{2} K_{0}+\frac{1}{2} \gamma_{(2)} t_{1}{ }^{2} t_{(1)}{ }^{2} K_{0} \\
+\alpha_{2} t_{(2)}{ }^{2} t_{1}{ }^{2} t_{(1)}{ }^{2} K_{0}+\frac{1}{2} \gamma_{(3)} t_{2}{ }^{2} t_{(2)}{ }^{2} t_{1}{ }^{2} t_{(1)}{ }^{2} K_{0} \\
+\alpha_{3} t_{(3)}{ }^{2} t_{2}{ }^{2} t_{(2)}{ }^{2} t_{1}{ }^{2} t_{(1)}{ }^{2} K_{0} \\
+\frac{1}{2} \gamma_{(4)} t_{3}{ }^{2} t_{(3)}{ }^{2} t_{2}{ }^{2} t_{(2)}{ }^{2} t_{1}{ }^{2} t_{(1)}{ }^{2} K_{0} \\
+\alpha_{5} t_{(4)}{ }^{2} t_{3}{ }^{2} t_{(3)}{ }^{2} t_{2}{ }^{2} t_{(2)}{ }^{2} t_{1}{ }^{2} t_{(1)}{ }^{2} K_{0} \\
K \downarrow_{2}=K_{0} t_{(1)} t_{1} t_{(2)}
\end{gathered}
$$

$$
\begin{aligned}
K \uparrow_{2}= & \alpha_{2} t_{(1)} t_{1} t_{(2)} K_{0}+ \\
+ & \frac{1}{2} \gamma_{3} t_{2}{ }^{2} t_{(2)} t_{1} t_{(1)} K_{0} \\
& +\alpha_{3} t_{(3)}{ }^{2} t_{2}{ }^{2} t_{(2)} t_{1} t_{(1)} K_{0}+\frac{1}{2} \gamma_{(4)} t_{3}{ }^{2} t_{(3)}{ }^{2} t_{2}{ }^{2} t_{(2)}{ }^{2} t_{1} t_{(1)} K_{0} \\
& +\alpha_{5} t_{(4)}{ }^{2} t_{3}{ }^{2} t_{(3)}{ }^{2} t_{2}{ }^{2} t_{(2)}{ }^{2} t_{1} t_{(1)} K_{0} .
\end{aligned}
$$

In these equations $\alpha$ is the cloud albedo, $\gamma$ is the scattering coefficient, $t$ is the transmissivity following absorption, $I_{0}$ is the solar constant, and $z$ is the zenith angle. For this study, a value of $1353 \mathrm{~W} \mathrm{~m}^{-2}$ (Paltridge and Platt, (1976)) is used for the solar constant.

Again, similar sets of equations are used for levels 2 and 3. Thus for level 2

$$
\left(\frac{d K^{*}}{d \sigma}\right)_{2}=\frac{K \downarrow_{1}-K \uparrow_{1}-K \downarrow_{3}+K \uparrow_{3}}{\sigma_{1}-\sigma_{3}}
$$

where

$$
\begin{gathered}
K \downarrow_{1}=t_{(1)} t_{1} K_{0} \\
K \uparrow_{1}=\frac{1}{2} \gamma_{(2)} t_{1} t_{(1)} K_{0}+\alpha_{2} t_{(2)} t_{1} t_{(1)} K_{0} \\
+\frac{1}{2} \gamma_{(3)} t_{2}{ }^{2} t_{(2)}{ }^{2} t_{1} t_{(1)} K_{0}+\alpha_{3} t_{(3)}{ }^{2} t_{2}{ }^{2} t_{(2)}{ }^{2} t_{1} t_{(1)} K_{0} \\
+\frac{1}{2} \gamma_{(4)} t_{3}{ }^{2} t_{(3)}{ }^{2} t_{2}{ }^{2} t_{(2)}{ }^{2} t_{1} t_{(1)} K_{0} \\
+\alpha_{5} t_{(4)}{ }^{2} t_{3}{ }^{2} t_{2}{ }^{2} t_{(2)}{ }^{2} t_{1} t_{(1)} K_{0} \\
K \downarrow_{3}=t_{(3)} t_{2} t_{(2)} t_{1} t_{(1)} K_{0}
\end{gathered}
$$




$$
\begin{aligned}
K \uparrow_{3}=\alpha_{3} t_{(3)} t_{2} t_{(2)} t_{1} t_{(1)} K_{0} & +\frac{1}{2} \gamma_{(4)} t_{3}{ }^{2} t_{(3)} t_{2} t_{(2)} t_{1} t_{(1)} K_{0} \\
& +\alpha_{5} t_{(4)}{ }^{2} t_{3}{ }^{2} t_{(3)} t_{2} t_{(2)} t_{1} t_{(1)} K_{0} .
\end{aligned}
$$

In the same way, the vertical gradient of net shortwave radiation for level 3 is specified by

$$
\left(\frac{d K^{*}}{d \sigma}\right)_{3}=\frac{K \downarrow_{2}-K \uparrow_{2}-K \downarrow_{5}+K \uparrow_{5}}{\sigma_{2}-\sigma_{5}}
$$

where

$$
\begin{gathered}
K \downarrow_{2}=t_{2} t_{(2)} t_{1} t_{(1)} K_{0} \\
K \uparrow_{2}=\frac{1}{2} \gamma_{(3)} t_{2} t_{(2)} t_{1} t_{(1)} K_{0}+\alpha_{3} t_{(3)}{ }^{2} t_{2} t_{(2)} t_{1} t_{(1)} K_{0} \\
+\frac{1}{2} \gamma_{(4)} t_{3}{ }^{2} t_{(3)}{ }^{2} t_{2} t_{(2)} t_{1} t_{(1)} K_{0} \\
+\alpha_{5} t_{(4)}{ }^{2} t_{3}{ }^{2} t_{(3)}{ }^{2} t_{2} t_{(2)} t_{1} t_{(1)} K_{0} \\
K \downarrow_{5}=t_{(4)} t_{3} t_{(3)} t_{2} t_{(2)} t_{1} t_{(1)} K_{0} \\
K \uparrow_{5}=\alpha_{5} t_{(4)} t_{3} t_{(3)} t_{2} t_{(2)} t_{1} t_{(1)} K_{0}
\end{gathered}
$$

To solve these equations requires knowledge of the albedo for each level, the scattering coefficient for each layer and the transmission and absorption by each of the layers and levels. As with the longwave radiation, the layers are used to account for the effects of the atmospheric gases on shortwave radiation. Ozone is assumed to absorb 3\% of the extraterrestrial flux in the top layer. Below this, layer scattering is handled in the manner recommended by Davies and Hay (1980). In this case the scattering is assumed to be a function of the pressure depth of the layer. Thus

$$
\gamma / 2=\left(\Delta p / p^{*}\right) \alpha_{R}=\Delta \sigma \alpha_{R}
$$

where $\Delta \sigma$ is the sigma thickness of the layer and $\alpha_{R}$ is calculated using Lacis and Hansen's (1974) expression,

$$
\alpha_{R}=0.28 /(1+6.43 \mu) \text {. }
$$

In this model $\mu$ is calculated from

$$
\mu=\sin \phi \sin \delta+\cos \phi \cos \delta \cos H
$$

where $\phi$ is latitude, $\delta$ is declination, and $H$ is the hour angle.

The absorption by each layer is assumed to be a function of the proportion of the total water vapor in the column that is found in the layer. Lacis and Hansen's (1974) equation,

$$
\begin{aligned}
a_{w}\left(u_{w} / \mu\right)= & 0.29\left(u_{w} / \mu\right) / \\
& {\left[1+14.15\left(u_{w} / \mu\right)^{0.635}+0.5925\left(u_{w} / \mu\right)\right], }
\end{aligned}
$$

requires that $u_{w}$ be expressed in units of millimeters. The optical depths calculated for the layers in the determination of the longwave emissivities are modified accordingly. In the mean profile $7 \%$ of the water vapor is in layer $2,29 \%$ in layer 3 and $64 \%$ in layer 4 . The absorptivities are adjusted to reflect this distribution. The transmission through a layer is given by

$$
t_{(i)}=1-\gamma_{(i)} / 2-a_{(i)} \text {. }
$$

Since the cloud reflection and absorption is calculated at the information levels, some values of estimates are available. The values used by Manabe and Strickler (1964) in their general circulation model are most appropriate for the information levels in this model. These values are reproduced in Table 4 . Note that the surface albedo level (level 5) is taken from Sellers' (1965) value for the ocean. If there are no clouds present at a level, there is no reflection or absorption $\left(\alpha_{i}=a_{i}=0\right)$. Thus transmission through any level is specified by

$$
t_{i}=1_{i}-\alpha_{i}-a_{i} \text {. }
$$

Figure 11 shows the net radiative heating on the June solstice generated by this scheme. This sample calculation reproduces the general pattern of radiative heating quite well, with the lowest level exhibiting the greatest diurnal range and the highest level the least. The results of this and other preliminary calculations produce heating and cooling rates comparable to some of those described by Newell, et al., (1974), and thus confirm the appropriateness of the use of this method for the radiative experiment.

\section{d. The radiative simulation}

Addition of the effects of the net radiation to the diabatic heating term makes it possible to test the conceptual hypothesis that diurnal oscillations in tropical cyclones are caused by variations in the radiative fluxes. The results of a simulation designed to test this hypothesis are presented in this section in an attempt to demonstrate that this is indeed the case. The solar radiation fluxes are calculated for a tropical cyclone at $20^{\circ} \mathrm{N}$ latitude on the June solstice. This date is used because no day-to-day variation in solar radiation is included in the model, and the period around the solstice exhibits the least day-to-day variation in the extraterrestrial flux during the Atlantic hurricane season. The values of the radiative fluxes are recalculated only every half hour. This is an attempt to capture the important changes in these terms without creating an unreasonable increase in the computational time required for the simulation. The initial conditions, boundary conditions and other model parameters are identical to those used for the basic simulation. For purposes of

TABLE 4. Cloud absorptivities and albedos.

\begin{tabular}{ccc}
\hline Level & Albedo & Absorptivity \\
\hline 1 & 0.21 & 0.005 \\
2 & 0.48 & 0.02 \\
3 & 0.69 & 0.035 \\
5 & 0.06 & - \\
\hline
\end{tabular}




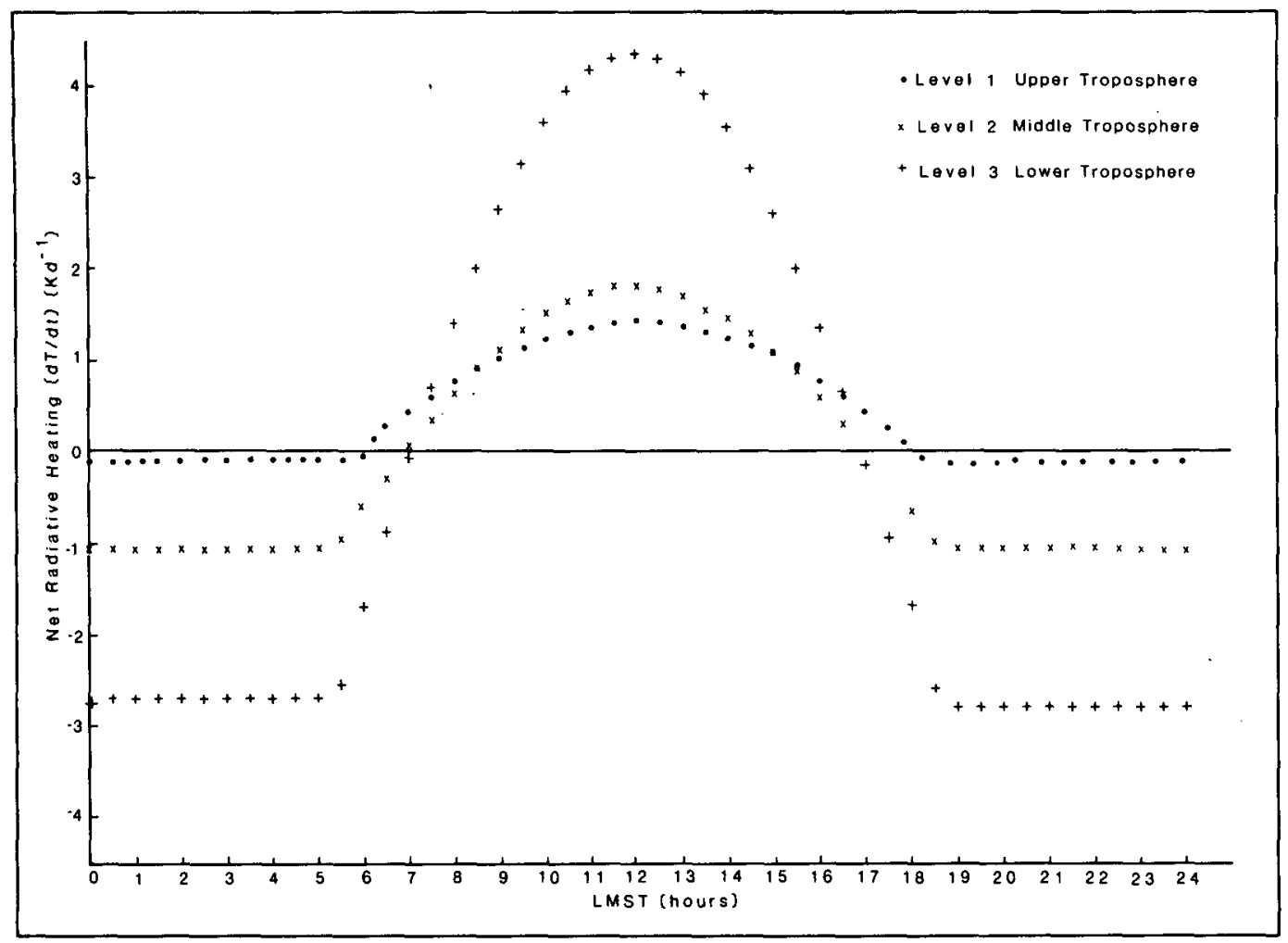

FIG. 11. Clear sky radiative heating rates at $20^{\circ} \mathrm{N}$ for the June solstice.

calculating the solar fluxes the simulation begins at 0000 LMST.

During the early hours of the radiative simulation, the pattern exhibited by the model tropical cyclone (initial dissipation, followed by intensification) is quite similar to that of the basic (nonradiative) simulation. This becomes evident if the maximum boundary wind speeds from the radiative simulation plotted in Fig. 12 are compared with those from the basic simulation plotted in Fig. 2. Similarly, the minimum surface pressures plotted in Figs. 13 and 3 exhibit this same pattern. In both cases the first ten hours of the simulations are nearly identical. Between 10 and $25 \mathrm{~h}$ the model cyclone generated during the radiative simulation strengthens rapidly, with the maximum wind speed reaching $45 \mathrm{~m} \mathrm{~s}^{-1}$ by $25 \mathrm{~h}$. This rate of intensification is much greater than that which occurred during the basic simulation. Between 25 and $35 \mathrm{~h}$ the intensity of the storm remains fairly constant, but the circulation becomes more well organized. After this time, a second period of rapid intensification occurs with the maximum winds reaching a speed of $73.6 \mathrm{~m} \mathrm{~s}^{-1}$ at $51 \mathrm{~h}$. This is followed by a gradual weakening that continues until approximately $80 \mathrm{~h}$, after which the maximum wind speed oscillates around a value of $60 \mathrm{~m} \mathrm{~s}^{-1}$.

Since the only alteration made to the model between the two simulations was the inclusion of the radiative fluxes, the different patterns of intensification can be attributed to this change. With the simulation starting at $0000 \mathrm{LMST}$, midnight, there is no incoming solar radiation during the early hours of the first day. Thus, the longwave fluxes are the only components contributing to the diabatic heating term in the thermodynamic equation. If the previously described conceptual model of the diurnal oscillations is accurate, the emission of longwave radiation to space by the cirrus clouds in the upper troposphere should produce intensification. However, these early hours of the simulation are dominated by effects caused by the introduction of friction, and thus these radiative fluxes appear to have little effect on the intensity of the model storm. This continues to be true, even after sunrise ( $06 \mathrm{~h}$ ) on the first day.

It is near sunset $(18 \mathrm{~h})$ on the first day that differences in the intensities of the model storms begin to become significant. Figure 14, which shows the differences in the maximum wind speeds between the two model tropical cyclones, and Fig. 15, which is a plot of the differences in the minimum sea level pressures, both clearly illustrate this. In both instances, the differences in intensity exhibit definite diurnal patterns during the early stages of the simulation. During this time, the loss of energy by the cloud tops at a rate of $3 \mathrm{~K}$ per day allows for greater convection and the model cyclone strengthens more rapidly than in the basic simulation. By $24 \mathrm{~h}$ (midnight) the model storm has ad- 


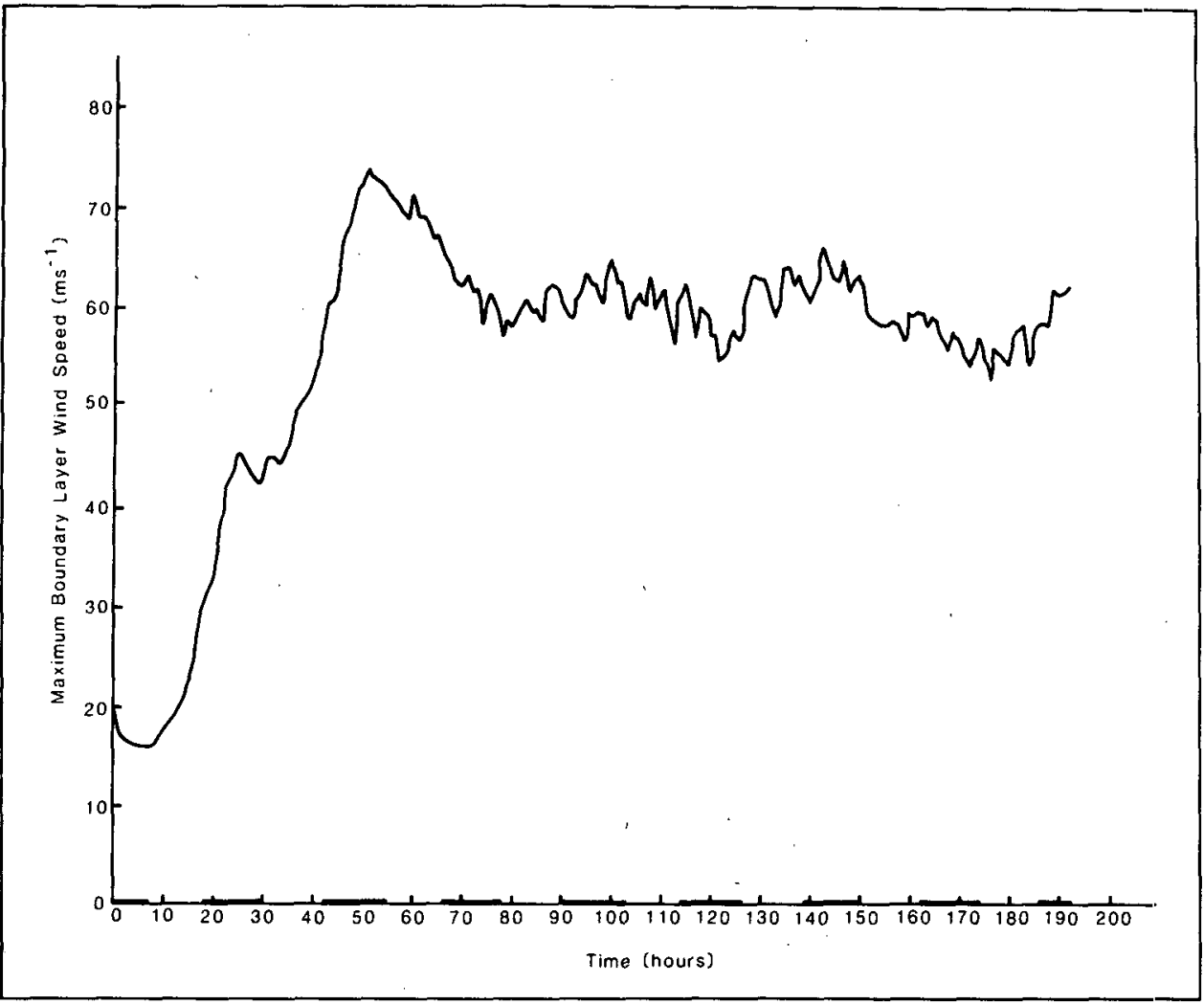

FIG. 12. Maximum boundary layer wind speeds vs time for radiative simulation. The thicker sections on the horizontal axis indicate the periods of darkness.

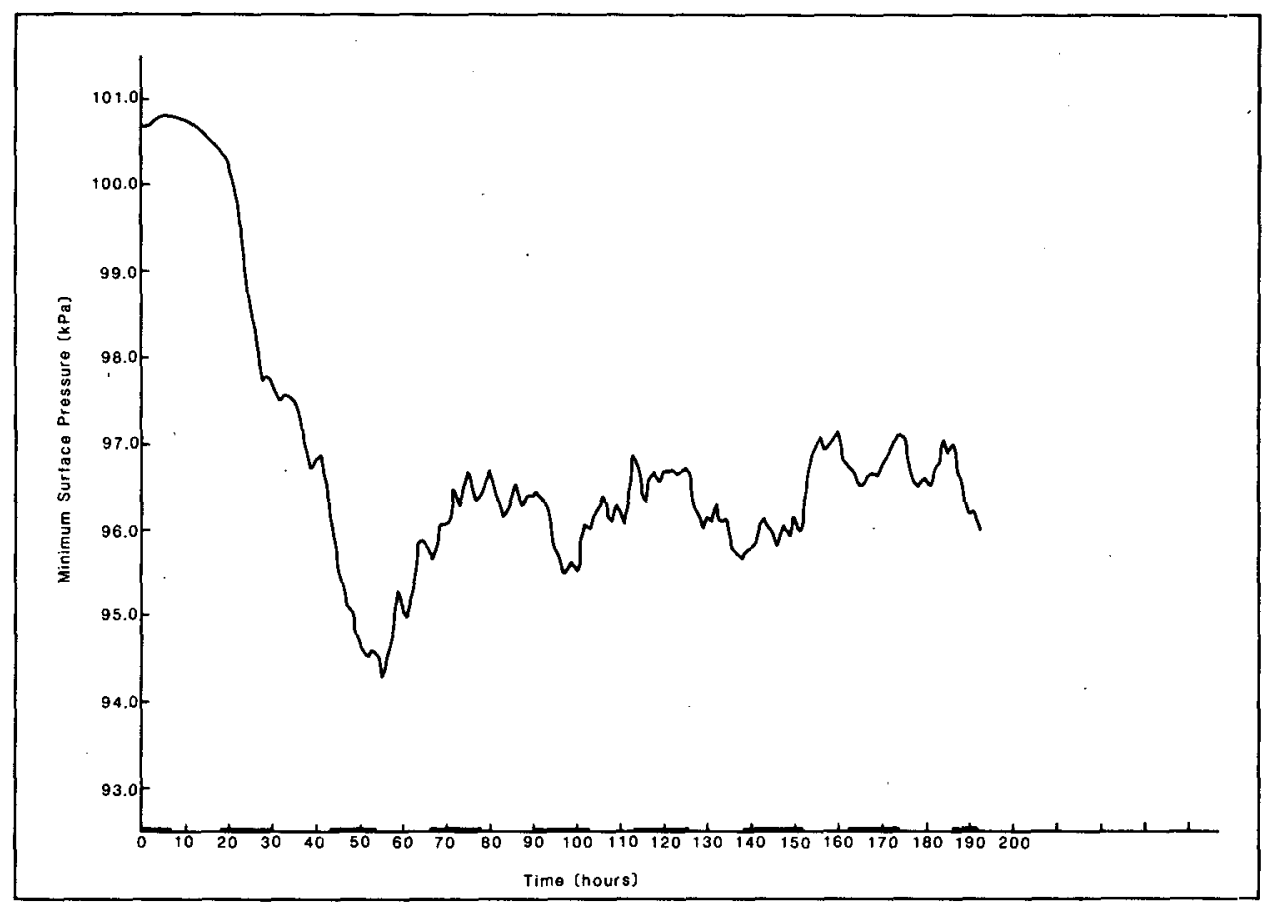

FIG. 13. As in Fig. 12 but for minimum surface pressure. 


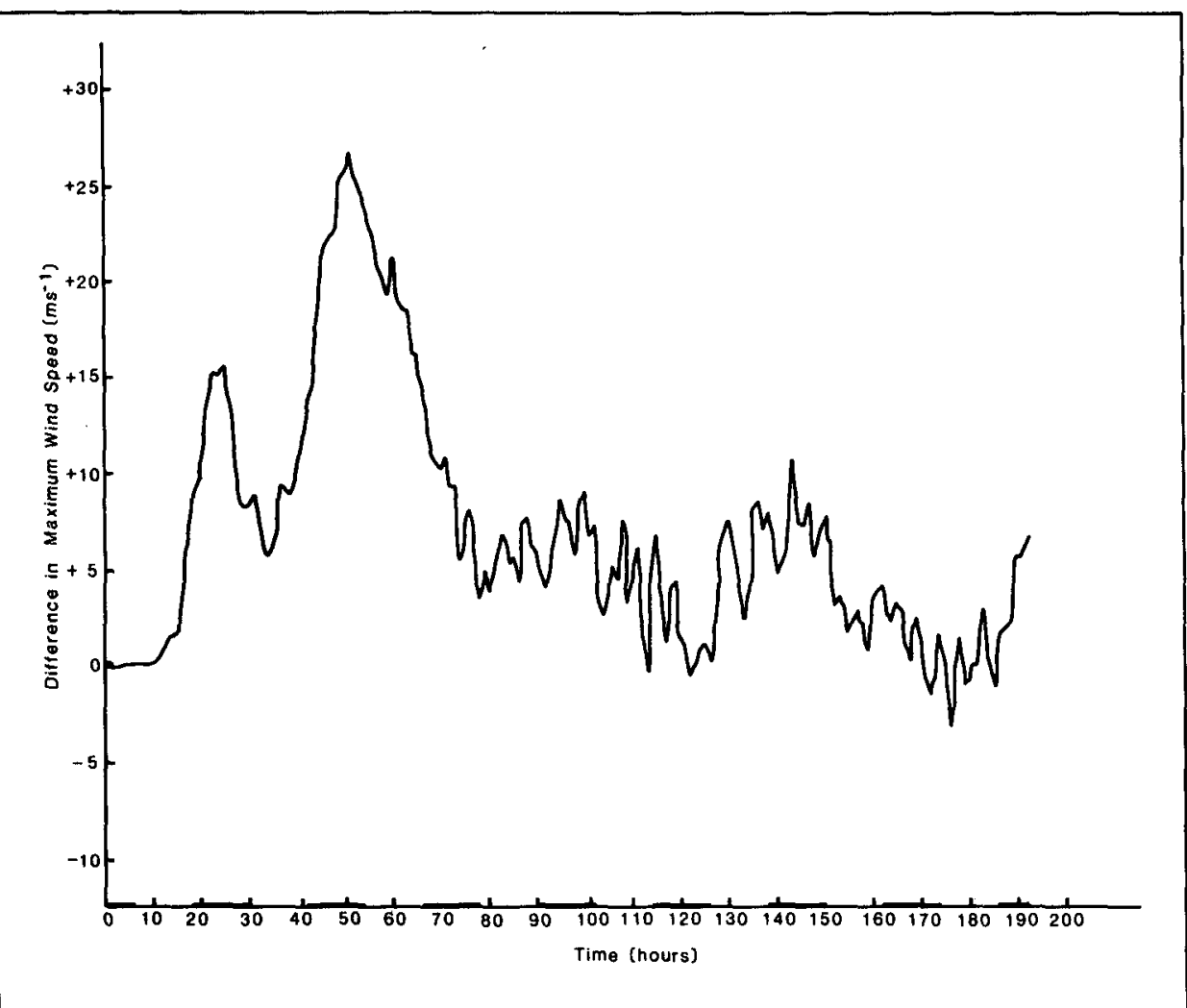

FIG. 14. Difference between maximum boundary layer wind speeds between the radiative and nonradiative simulations. The thicker sections on the horizontal axis indicate the periods of darkness.

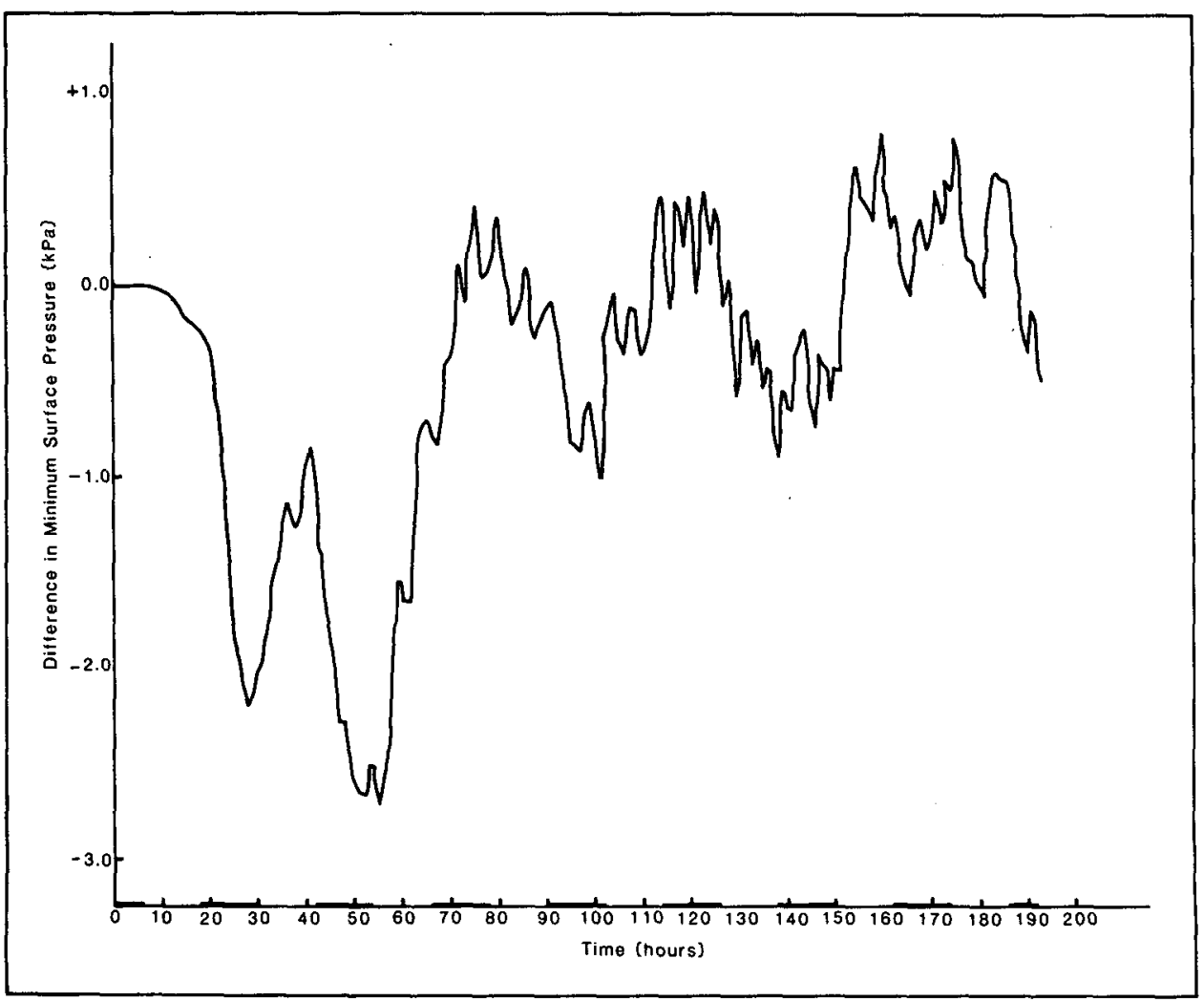

FIG. 15. As in Fig. 14 but for the minimum surface pressures. 
justed to this situation and the maximum wind speed remains fairly constant until $36 \mathrm{~h}$. During this period the storm becomes more well organized and the radius of maximum wind moves consistently closer to the center of the storm. After $36 \mathrm{~h}$ the system resumes intensification at a fairly normal rate. Nightfall on the second day brings on another period of more rapid strengthening as the cloud top cooling augments the normal convection. By midnight of the second day (48 h) the maximum wind speeds are almost $70 \mathrm{~m} \mathrm{~s}^{-1}$. This value is greater than that reached in the basic simulation and clearly demonstrates the ability of radiative cooling of the cloud tops (approximately 1-2 $\mathrm{K}$ in areas of active convection) to increase the intensity of a tropical cyclone. This is similar to the finding of Kurihara and Tuleya (1981) on the effects of radiation on the development of a tropical cyclone.

The model hurricane is unable to sustain this intensity as the increased latent heating begins to offset the radiative cooling. After sunrise on day $3(54 \mathrm{~h})$ solar heating further contributes to this weakening. From this time until the end of the simulation the strength of the model storm oscillates around a maximum wind speed of $60 \mathrm{~m} \mathrm{~s}^{-1}$. The diurnal effects of the radiative fluxes, which are quite apparent for the first three days, become much less obvious. This is consistent with the empirical studies (e.g., Browner et al., 1977) that found an inverse relationship between the diurnal oscillation and storm intensity. In fact, inspection of Figs. 12 and 13 reveals that the oscillation exhibits a period of 48 $h$. This probably results from the interaction between the radiative fluxes and the internal dynamics of the model storm. By the later stages of the simulations, the intensities of the two model storms are about the same. This reflects the use of the same initial and boundary values in both cases.

As is the case in the basic simulation, the structure of the storm continues to evolve after $72 \mathrm{~h}$. After this time, spiral bands begin to develop in the outflow. Because the storm is more intense than the one produced during the basic simulation, the outflow in the spiral bands is stronger. Also, because the longwave cooling of the cloud tops at night cause the model cyclone to intensify more quickly, the spiral bands appear at an earlier point in the simulation. As Fig. 16 shows, by $72 \mathrm{~h}$ the first spiral band is well developed and is moving away from the center of the system. This is a full day before the spiral bands appeared in the basic simulation. Thus, inclusion of the radiative fluxes seems to lead to a more rapid intensification and development of the asymmetrical features, such as the spiral bands. The earlier appearance of the spiral bands also suggests that the development of asymmetries in the structure of the storm is a function of the intensity of the model hurricane.

Analysis of the cloudiness in the top layer reveals no apparent diurnal oscillation of the areal extent of the cirrus canopy. The proximity of the outer boundary to the core of the storm is the probable reason that no oscillation is discernible. As the model storm intensifies, the upper-level outflow develops and rapidly transports the cirrus to the edge of the model domain. Thus, even with the introduction of the radiative fluxes, the outward transportation of the cirrus maintains the areal extent of the canopy. In fact, since the storm intensifies more rapidly when the radiative fluxes are included, the outflow is even stronger than in the basic simulation. In an attempt to determine if the radiative fluxes affect convection and rainfall rates, the total latent heating rate over the entire storm is presented in Fig. 17. Higher latent heating values result from increased convection and are directly correlated with higher rainfall rates.

For the first $24 \mathrm{~h}$ the latent heating increases steadily. During this period the low-level convergence, produced by the introduction of friction in the first time step, generates rising motion. This rising motion initiates convection, which becomes more pronounced as the intensification process begins. The effects of the radiative fluxes on this process first become evident at $36 \mathrm{~h}$ (noon LMST on the second day). Absorption of solar radiation at the cloud tops produces warming in the upper troposphere, thus reducing the lapse rate and slowing convection. This is seen in Fig. 17 as a relatively steady release of latent energy, which is maintained by the well-developed low-level inflow. As shown in Figs. 12 and 13 , the intensification process slowed considerably during this period.

After sunset, approximately $42 \mathrm{~h}$ into the simulation, the emission of longwave radiation by the cloud tops produces cooling in the upper layer of the model. This increases the lapse rate and increases convection. The increased convection releases greater amounts of latent energy, as seen by the rapid increase in latent heating between 36 and $48 \mathrm{~h}$. This latent heating strengthens the warm core of the model storm and leads to a period of rapid intensification. After sunrise on the third day, approximately $54 \mathrm{~h}$ into the simulation, the absorption of solar radiation at the cloud tops again warms the upper layer, slowing convection and the release of latent energy. This is seen in Fig. 17 as a decline in the rate of latent heating between 48 and $60 \mathrm{~h}$, noon LMST on day 3 . This decline is significant evidence for the influence of the radiative fluxes, since in the basic simulation this time was a period of rapid intensification. After nightfall on day 3, approximately $66 \mathrm{~h}$, upperlevel cooling increases convection and latent heating. Thus, the pattern of stronger convection during the night and weaker lifting during the day exists through day $3,72 \mathrm{~h}$ into the simulation.

After $72 \mathrm{~h}$ the pattern changes phase; the greatest heating occurs around noon LMST each day and the least at midnight LMST. While initially puzzling, the reason for this change is fairly obvious. As shown in Fig. 16, the first spiral band begins developing about $72 \mathrm{~h}$ into the simulation. After this time convection 


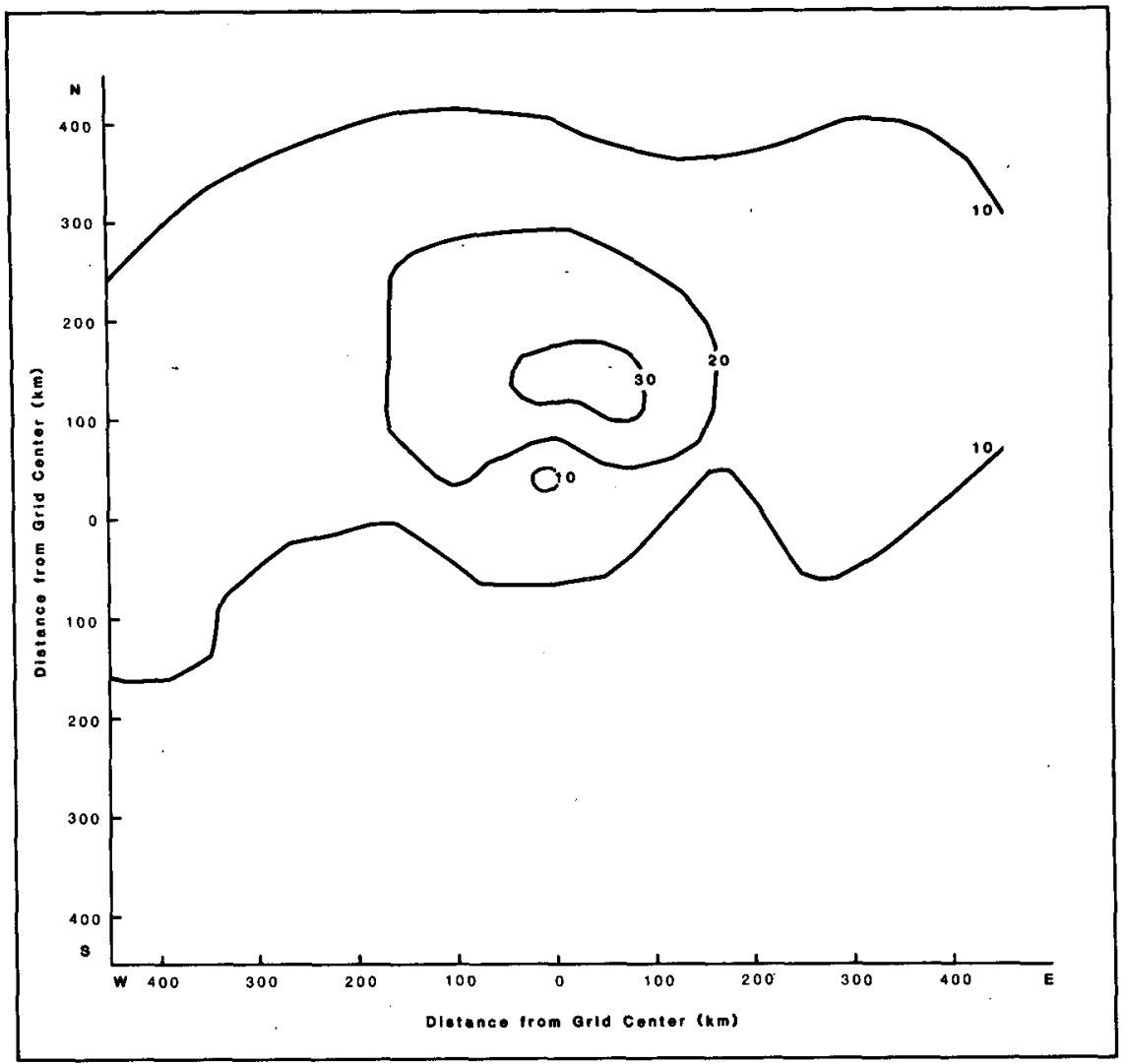

FIG. 16. Upper tropospheric isotachs at $72 \mathrm{~h}$ for the radiative simulation.

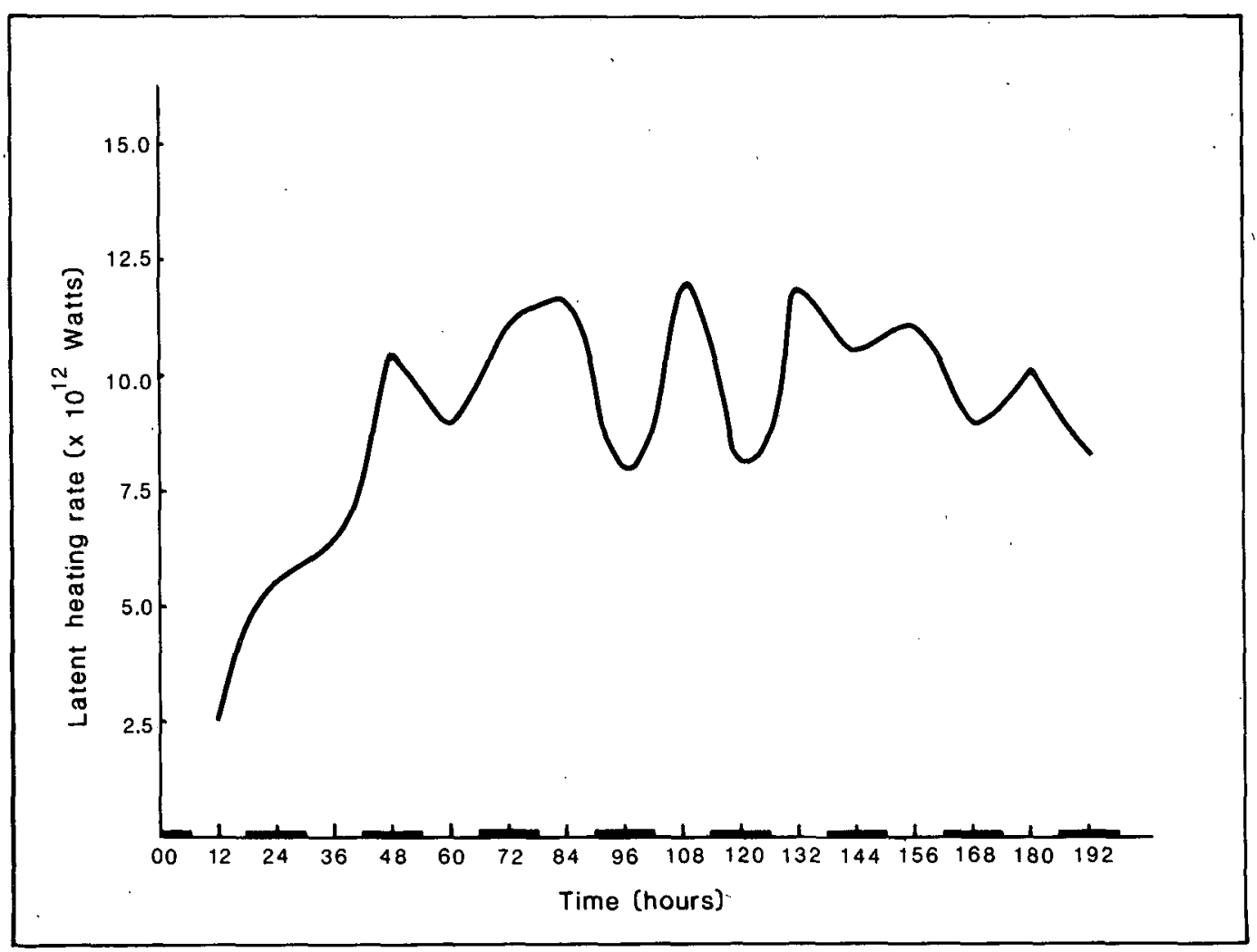

FIG. 17. Total latent heating rate of the entire storm for the radiative simulation. The thicker sections on the horizontal axis indicate the periods of darkness. 
and the rate of latent heating become primarily determined by the appearance of these features. As a new band develops, there is a substantial increase in convection, which gradually diminishes as the band moves away from the center of the storm and weakens. Each time a new band forms, the convection reintensifies and the rate of latent heating increases. Thus, in the weaker stages, radiation directly affects convection, as seen in the rates of latent heating during the first three days of the simulation. In the later, stronger stages of the storm the internal dynamics of the tropical cyclone become more important and the radiative influences become much less evident.

While an examination of the cloud cover in the top layer of the model reveals no apparent diurnal oscillation of the cirrus canopy, the pattern of latent heating discussed previously provides indirect evidence that such an oscillation would be likely to appear if the horizontal domain of the model were expanded. Support for this assertion is provided by the work of Merritt and Wexler (1967) on outflow rates for hurricanes. The higher latent heating rates coincide with periods of strong convection and production of cirrus. In the first three days of the simulation this occurred around midnight LMST. Merritt and Wexler (1967) determined that it took $12-18 \mathrm{~h}$ for air near the center of a hurricane to reach the outer regions of the circulation in the upper layers of the storm. This means that the maximum areal extent of the cirrus should occur sometime between 1200 and 1800 LMST (12-18 h after its generation at midnight). This compares well with the period of maximum extent observed by Browner et al. (1977), which was from 1200-1900 LMST. Conversely, with the minimum convection occurring around noon LMST during the first three days of the simulation, the minimum areal extent should occur between 0000 and 0600 LMST on the next day. This is within the period of minimum extent observed by Browner et al., which ranged from 2330-0700 LMST.

Thus, while the diurnal oscillation of the cirrus canopy is not directly simulated by this version of the model, the pattern of latent heating and prior empirical work on the outflow rates in the upper part of the storm indicate that this oscillation would appear if the horizontal domain were larger. More importantly, the results presented from the simulation with the radiative fluxes, included in the diabatic heating term of the thermodynamic energy equation, clearly demonstrate that these fluxes are capable of inducing a diurnal oscillation into the intensification process during the weaker stages of the storm.

Inspection of the vertical velocities reveals no evidence of the pattern proposed by Gray and Jacobson (1977) to account for the diurnal oscillation. This is possibly due to the previously mentioned fact that cirrus quickly covers the entire domain of the model and remains throughout the simulation. Thus, significant clear areas, which comprise a key aspect of Gray and Jacobson's explanation of the diurnal oscillations, are not found within the model's domain. Since subsidence in clear areas is an important component in the mechanism proposed by Lajoie and Butterworth (1984) to explain the daytime oscillation, the model is also unable to replicate this feature. Therefore, it is not possible to attribute the shorter-period fluctuations of intensity in Fig. 12 to that mechanism.

\section{Summary and conclusions}

The research reported in this paper consists of three parts. First, a conceptual model is presented that includes the physical mechanisms necessary to account for the diurnal changes observed in tropical cyclones. In this model the primary cause of the diurnal oscillations is attributed to the net radiation at the cloud tops. The absorption of solar radiation during the day creates slightly warmer conditions at the cloud tops and thus a slight increase in the stability. At night lower temperatures generate a slight decrease in the stability. Since a tropical cyclone often exists in a condition of saturated neutrality, the changes in stability generate increased convection at night and decreased convection during the day. The increased convection at night produces more precipitation, greater latent heating, more rising motion and slightly lower surface pressures. The decreased convection during the day generates less precipitation, reduced latent heating, less rising motion and higher pressures. In addition, the changes in convection affect the development of the cirrus canopy and its areal extent. As a storm develops and the radiative fluxes become a smaller proportion of the total energy budget, the diurnal fluctuations become less pronounced. The increased subsidence in clear areas at night proposed by Gray and Jacobson (1977) augments the cooling of the cloud tops and also forms a part of Lajoie and Butterworth's (1984) explanation of a shorter-period daytime oscillation.

In the second portion of this investigation, the numerical model used for the simulations is described. This model is developed from the basic equations of motion written in the $(x, y, \sigma, t)$ coordinate system. A staggered grid with a $25 \mathrm{~km}$ interval between points and a $37.5 \mathrm{sec}$ time increment provide economical, yet detailed, resolution of the structure of the model storm. The choice of four vertical layers facilitates the later incorporation of the radiative fluxes. The basic model simulates realistically the development of a strong hurricane from the initially weak tropical depression. Additionally, the model successfully reproduces the major features associated with an intense tropical cyclone. The model storm exhibits strong, cyclonic, low-level inflow and weaker, anticyclonic, upper-level outflow. Spiral rainbands develop despite an initially symmetrical circulation and evidence of an eye is seen in the vertical motion around the center of the system. Thus, 
it is clear that the model storm possesses all of the major features of a tropical cyclone.

Finally, after the addition of explicit radiative fluxes to the basic model, a discussion is presented of an attempt to reproduce the diurnal oscillations of tropical cyclones. The diabatic heating is computed using the vertical gradient of net radiation across the level for which the heating is desired. Both clear sky and cloud effects are included in the calculations. The longwave emissivities are derived from Staley and Jurica's (1970) tables, while shortwave attenuation is handled in the manner suggested by Davies and Hay (1980). The radiative simulation begins at 0000 LMST and all values for the shortwave fluxes are calculated for the date of the June solstice. Evidence from the surface pressures and wind speeds indicate that, while the final structure of the storm is not greatly affected by the radiative fluxes, the development is influenced by these processes. This is consistent with the conceptual model that states that the effects of radiation decline as the storm intensifies. Perhaps the most disappointing result is the inability to show explicitly the oscillation in the areal extent of the cirrus canopy within the current model domain. However, examination of the total latent heating rates clearly indicates that, in the early stages of the simulation, convection is affected by the radiative fluxes in the manner proposed. Combining this fact with the empirical work of Merritt and Wexler (1967) on outflow, it seems probable that, if the model domain is expanded, the expected oscillations in the cirrus canopy will be present. Thus, the results seem to validate this conceptual model as a mechanism capable of generating the diurnal oscillations that have been observed in tropical cyclones. Inspection of the results of the simulations reveals no evidence of the processes described by Gray and Jacobson (1977) and Lajoie and Butterworth (1984). However, this is probably caused by the relatively small domain of the model, which does not allow for the development of the significant clear areas that are a key part of these processes.

The most important conclusion to be drawn from this work is that the magnitudes of the fluxes of shortwave and longwave radiation are sufficient to induce a diurnal oscillation in the rate of latent heating of the model tropical cyclone during its weaker stages. Several other important results are implicit in this finding. First, for weaker tropical cyclones the greatest amounts of convection and rainfall should occur at night when the atmosphere is slightly less stable. Lesser amounts of convection and rainfall occur during the day because of slightly more stable conditions. Second, since the tropical cyclone possesses a warm core, the increased latent heating at night produces intensification. Thus, if all other factors remain constant, a weak tropical cyclone should be more intense at night than it is during the day. All of these things have important implications for forecasters dealing with tropical cyclones.
Additionally, these findings provide support for the empirical studies that originally suggested diurnal oscillations might exist in tropical cyclones. While unable in its present form to reproduce diurnal oscillations in its cirrus canopy, the results strongly suggest that the pattern reported by Browner et al. (1977) and Steranka et al. (1984) do exist, and that the diurnal oscillation of net radiation can generate a similar pattern. By demonstrating a diurnal effect on surface pressures, the results from the model also provide support for Hebert and Jarvinen's (1977) findings.

While providing strong evidence that diurnal oscillations exist in tropical cyclones, this research represents only a beginning. Several improvements to the model that would enhance its capabilities are now evident. First, the horizontal domain needs to be expanded. By moving the lateral boundary farther from the core of the circulation, it should be possible to reproduce the diurnal oscillations in the cirrus canopy. This would provide further proof for the existence of these oscillations, and allow for a better test of Gray and Jacobson's (1977) and Lajoie and Butterworth's (1984) proposed mechanisms. An improvement in the vertical resolution of the model might be attained by reorganizing the vertical structure and by making the stratosphere a separate layer. This might also remove the need for the adjustment of the initial temperature in the upper layer which was made by these experiments. Numerous improvements to the manner in which the radiative fluxes are calculated by the model are also possible. In particular, longwave emissivities could be improved by including vapor pressure-broadened continuum effects described by Cox (1973). A more sophisticated approach might also include a matrix of emissivities in place of the individual values for each of the layers used in this study.

Because of the computer resources required for each simulation, the model has only been used to simulate one date during the hurricane season for a storm at one latitude. Further simulations need to be made for different times during the year and for storms at different latitudes. This would provide information on any temporal or spatial variability in the magnitudes of the oscillations. The results from these simulations could be compared with data gathered on similar tropical cyclones in order to learn more about the nature of diurnal oscillations in tropical cyclones.

Determination of the nature and causes of these oscillations holds great significance for forecasters. Many techniques used for predicting movement and intensity of these systems are highly dependent upon satellite imagery. One such scheme, designed by Dvorak (1975), forecasts changes in intensity from the cloud patterns observed during the past $24 \mathrm{~h}$. Obviously, in order to make an accurate forecast, it is necessary to separate the normal, diurnal variations in the cirrus canopy from changes which indicate more significant developments. Thus, the results from the simulations per- 
formed in this study represent another step in making this possible.

Acknowledgments. The author would like to thank Dr. J. N. Rayner for his useful comments during the construction of the model and Dr. A. J. Arnfield for his suggestions on the inclusion of the radiative fluxes. He would also like to thank the Department of Geography at the Ohio State University for providing the computer funds for this project.

\section{REFERENCES}

Anthes, R. A., 1972: Development of asymmetries in a three-dimensional numerical model of the tropical cyclone. Mon. Wea. Rev., 100, 461-476.

- S. L. Rosenthal and J. W. Trout, 1971: Preliminary results from an asymmetric model of the tropical cyclone. Mon. Wea. Rev., 99, 744-758.

Browner, S. P., W. L. Woodley and C. G. Griffith, 1977: Diurnal oscillation of the area of cloudiness associated with tropical storms. Mon. Wea. Rev., 105, 856-864.

Cox, S. K., 1973: Infrared heating calculations with a water vapor pressure broadened continuum. Quart. J. Roy. Meteor. Soc., 99, 669-679.

Davies, J. A., and J. E. Hay, 1980: Calculation of the solar radiation incident on a horizontal surface. Proc. First Canadian Solar Radiation Data Workshop J. E. Hay and T. K. Won, Eds., 3258.

Dvorak, V. F., 1975: Tropical cyclone intensity analysis and forecasting from satellite imagery. Mon. Wea. Rev., 103, 420-430.

Garstang, M., 1979: The tropical atmospheric boundary layer. Role in the formation and maintenance of hurricanes. Aust. Meteor. Mag., 27, 229-248.

Gray, W. M., and R. W. Jacobson, Jr., 1977: Diurnal variation of deep cumulus convection. Mon. Wea. Rev., 105, 1171-1188.

Hebert, P. J., and C. L. Jordan, 1959: Mean soundings for the Gulf of Mexico area. Mon. Wea. Rev., 87, 217-221.

- , and B. R. Jarvinen, 1977: National Hurricane Center (NHC) diagnostic calculations on tropical cyclones. Preprints 11th Tech. Conf. on Hurricanes and Tropical Meteorology, Amer. Meteor. Soc., 247-252.

Hewson, E. W., 1943: The reflection, absorption, and transmission of solar radiation by fog and cloud. Quart. J. Roy..Meteor. Soc., 69, 47-62.
Hitchfeld, W., and J. T. Houghton, 1961: Radiative transfer in the lower stratosphere due to the 9.6 micron biand of ozone. Quart. J. Roy. Meteor., Soc., 87, 562-577.

Jones, R. W., 1977: A nested grid for a three-dimensional model of a tropical cyclone. J. Atmos. Sci., 34, 1528-1553.

Kurihara, Y., 1968: Note on finite difference expressions for the hydrostatic relation and pressure gradient force. Mon. Wea. Rev., 96, 654-656.

_- and R. E. Tuleya, 1981: A numerical study on the genesis of a tropical storm. Mon. Wea. Rev., 109, 1629-1653.

Lacis, A. A., and J. E. Hansen, 1974: A parameterization for the absorption of solar radiation in the earth's atmosphere. J. Atmos. Sci., 31, 118-133.

Lajoie, F. A., and I. J. Butterworth, 1984: Oscillation of high-level cirrus and heavy precipitation around Australian region tropical cyclones. Mon. Wea. Rev., 112, 535-544.

Manabe, S., and R. F. Strickler, 1964: Thermal equilibrium of the atmosphere with a convective adjustment. J. Atmos. Sci., 21, 361-385.

Matsuno, T., 1966: Numerical integration of the primitive equations by a simulated backard difference method. J. Meteor. Soc. Japan, Ser. 2, 44, 76-84.

Merritt, E. S., and R. Wexler, 1967: Cirrus canopies in tropical storms. Mon. Wea. Rev., 95, 111-120.

Newell, R. E., J. W. Kidson, D. G. Vincent and G. J. Boar, 1974: The General Circulation of the Tropical Atmosphere and Interactions with Extratropical Latitudes, Vol. 2. MIT Press, 371 pp.

Paltridge, G. W., and C. M. R. Platt, 1976: Radiative Processes in Meteorology and Climatology. Elsevier Scientific, 318 pp.

Phillips, N. A., 1957: A coordinate system having some special advantages for numerical forecasting. J. Meteor., 14, 184-185.

Reed, R. J., 1983: The diurnal variation of precipitation in the tropics. Preprints 1st. Int. Conf. Southern Hemisphere Meteorology, San Jose, Brazil, Amer. Meteor. Soc., 312-319.

Roll, H. U., 1965: Physics of the Marine Atmosphere. Academic Press, $426 \mathrm{pp}$.

Sellers, W. D., 1965: Physical Climatology. University of Chicago Press, $272 \mathrm{pp}$.

Staley, D. O., and G. M. Jurica, 1970: Flux emissivity tables for water vapor, carbon dioxide and ozone. J. Appl. Mfeteor., 9, 365-372.

Steranka, J., E. B. Rodgers and R. C. Gentry, 1984: The diurnal variation of Atlantic Ocean tropical cyclone distribution inferred from geostationary satellite infrared measurements. Mon. Wea. Rev., 112, 2338-2344.

Wetherald, R. T., and S. Manabe, 1980: Cloud cover and climate sensitivity. J. Atmos. Sci., 37, 1485-1510. 